\title{
THE LOCAL HOSTS OF TYPE Ia SUPERNOVAE
}

\author{
James D. Neill ${ }^{1}$, Mark Sullivan ${ }^{2}$, D. Andrew Howell ${ }^{3,4},{\text { Alex } \text { Conley }^{5,6} \text {, Mark Seibert }}^{7}$, D. Christopher Martin ${ }^{1}$, \\ Tom A. Barlow ${ }^{1}$, Karl Foster ${ }^{1}$, Peter G. Friedman $^{1}$, Patrick Morrissey ${ }^{1}$, Susan G. NefF ${ }^{8}$, David Schiminovich ${ }^{9}$, \\ Ted K. Wyder ${ }^{1}$, Luciana Bianchi ${ }^{10}$, José Donas $^{11}$, Timothy M. Heckman ${ }^{12}$, Young-Wook LeE ${ }^{13}$, Barry F. Madore ${ }^{7}$, \\ Bruno Milliard ${ }^{11}$, R. Michael Rich ${ }^{14}$, and Alex S. Szalay ${ }^{12}$ \\ ${ }^{1}$ California Institute of Technology, 1200 E. California Blvd., Pasadena, CA 91125, USA \\ ${ }^{2}$ University of Oxford, Denys Wilkinson Building, Keble Road, Oxford, OX1 3RH, UK \\ ${ }^{3}$ Las Cumbres Observatory Global Telescope Network, 6740 Cortona Dr., Suite 102, Goleta, CA 93117, USA \\ ${ }^{4}$ Department of Physics, University of California, Santa Barbara, Broida Hall, Mail Code 9530, Santa Barbara, CA 93106-9530, USA \\ ${ }^{5}$ Department of Astronomy and Astrophysics, University of Toronto, 50 St. George Street, Toronto, ONM5S3H8, Canada \\ ${ }^{6}$ Center for Astrophysics and Space Astronomy, University of Colorado, Boulder, CO 80309-0389, USA \\ ${ }^{7}$ The Observatories of the Carnegie Institute of Washington, 813 Santa Barbara Street, Pasadena, CA, 91101, USA \\ ${ }^{8}$ Laboratory for Astronomy and Solar Physics, NASA Goddard Space Flight Center, Greenbelt, MD 20771, USA \\ ${ }^{9}$ Department of Astronomy, Columbia University, New York, NY 10027, USA \\ ${ }^{10}$ Center for Astrophysical Sciences, Johns Hopkins University, 3400 N. Charles St., Baltimore, MD 21218, USA \\ ${ }^{11}$ Laboratoire d'Astrophysique de Marseille, BP 8, Traverse du Siphon, 13376 Marseille Cedex 12, France \\ 12 Department of Physics and Astronomy, Johns Hopkins University, Homewood Campus, Baltimore, MD 21218, USA \\ ${ }^{13}$ Center for Space Astrophysics, Yonsei University, Seoul 120-749, Republic of Korea \\ ${ }^{14}$ Department of Physics and Astronomy, University of California, Los Angeles, CA 90095, USA \\ Received 2009 July 13; accepted 2009 November 4; published 2009 December 4
}

\begin{abstract}
We use multi-wavelength, matched aperture, integrated photometry from the Galaxy Evolution Explorer (GALEX), the Sloan Digital Sky Survey, and the RC3 to estimate the physical properties of 166 nearby galaxies hosting 168 well-observed Type Ia supernovae (SNe Ia). The ultraviolet (UV) imaging of local SN Ia hosts from GALEX allows a direct comparison with higher-redshift hosts measured at optical wavelengths that correspond to the rest-frame UV. Our data corroborate well-known features that have been seen in other SN Ia samples. Specifically, hosts with active star formation produce brighter and slower SNe Ia on average, and hosts with luminosity-weighted ages older than 1 Gyr produce on average more faint, fast, and fewer bright, slow SNe Ia than younger hosts. New results include that in our sample, the faintest and fastest SNe Ia occur only in galaxies exceeding a stellar mass threshold of $\sim 10^{10} M_{\odot}$, leading us to conclude that their progenitors must arise in populations that are older and/or more metal rich than the general SN Ia population. A low host extinction subsample hints at a residual trend in peak luminosity with host age, after correcting for light-curve shape, giving the appearance that older hosts produce less-extincted SNe Ia on average. This has implications for cosmological fitting of SNe Ia, and suggests that host age could be useful as a parameter in the fitting. Converting host mass to metallicity and computing ${ }^{56} \mathrm{Ni}$ mass from the supernova light curves, we find that our local sample is consistent with a model that predicts a shallow trend between stellar metallicity and the ${ }^{56} \mathrm{Ni}$ mass that powers the explosion, but we cannot rule out the absence of a trend. We measure a correlation between ${ }^{56} \mathrm{Ni}$ mass and host age in the local universe that is shallower and not as significant as that seen at higher redshifts. The details of the age- ${ }^{56} \mathrm{Ni}$ mass correlations at low and higher redshift imply a luminosity-weighted age threshold of $\sim 3$ Gyr for SN Ia hosts, above which they are less likely to produce SNe Ia with ${ }^{56} \mathrm{Ni}$ masses above $\sim 0.5 M_{\odot}$.
\end{abstract}

Key words: galaxies: evolution - supernovae: general

Online-only material: color figures

\section{INTRODUCTION}

A fundamental motivation for studying Type Ia supernovae (SNe Ia) is to arrive at a physical explanation for the observed fact that these objects have well-behaved and calibratable explosions (Phillips 1993). This property gives cosmographers a tool for measuring universal expansion and provided the first direct observational evidence for cosmic acceleration (Riess et al. 1998; Perlmutter et al. 1999). Knowing the physical process that generates $\mathrm{SNe}$ Ia provides strong constraints on the nature of the SN Ia progenitor. This, in turn, allows us to predict the evolution of the SN Ia population (e.g., Howell et al. 2007). This results in tighter control of the systematic errors arising from population evolution with redshift, an important uncertainty in measuring cosmological parameters (e.g., Astier et al. 2006; A. Conley et al. 2010, in preparation). A wellconstrained physical model for SNe Ia would have further utility in tracing high-redshift star formation and for predicting the effects of SNe Ia on the chemical enrichment of their host galaxies (e.g., Kobayashi \& Nomoto 2007).

In order to calibrate $\mathrm{SNe}$ Ia and make them useful for cosmology, their intrinsic variation must be measured and accounted for. The peak absolute magnitude of a given SN Ia is a strong function of its initial decline rate (Phillips 1993), and a weaker function of its peak color (Riess et al. 1996; Tripp 1998; Tripp \& Branch 1999; Parodi et al. 2000). These empirical correlations reduce the intrinsic variation of $\sim 1 \mathrm{mag}$ in the $B$ band to $\sim 0.1 \mathrm{mag}$ and thus provide the accurate luminosity distance estimates required for measuring cosmological parameters (e.g., Riess et al. 1996; Tonry et al. 2003; Guy et al. 2005; Prieto et al. 2006; Jha et al. 2007; Conley et al. 2008). The goal of SN Ia progenitor studies is to use these measures of SN Ia intrinsic variation to explore trends with host properties that could shed light on the underlying 
population that produces SNe Ia (Phillips 1993; Hamuy et al. 1995, 2000; Howell 2001; Gallagher et al. 2005; Sullivan et al. 2006; Gallagher et al. 2008).

Since SN Ia explosions are powered by the radioactive decay of ${ }^{56} \mathrm{Ni}$, it is widely accepted that the intrinsic variation in $\mathrm{SN}$ Ia brightness and decline rate is primarily driven by the amount of ${ }^{56} \mathrm{Ni}$ present in the $\mathrm{SN}$ explosion, with more luminous and slower declining explosions being powered by more ${ }^{56} \mathrm{Ni}$ (Truran et al. 1967; Colgate \& McKee 1969). One avenue of exploration would be to connect this theoretical idea to the progenitor population of SNe Ia by comparing host galaxy properties with the measures of SN Ia light-curve variation. This requires a mechanism that varies the amount of ${ }^{56} \mathrm{Ni}$ as a function of some property of the host galaxy.

Timmes et al. (2003) presented a model relating progenitor metallicity to produced SN Ia ${ }^{56} \mathrm{Ni}$ mass for a constant (solar) $\mathrm{O} / \mathrm{Fe}$ ratio. This model was expanded and tested by Howell et al. (2009, hereafter H09) using an intermediate redshift $(0.2<z<0.75)$ sample of SNe Ia. They used spectral energy distribution (SED) fitting of optical integrated host photometry to infer the host stellar masses for their sample. These masses were used as a proxy for host metallicities through the Tremonti et al. (2004) mass-metallicity relation. They estimated the peak bolometric luminosity and rise time from the SN Ia photometry to calculate the required mass of ${ }^{56} \mathrm{Ni}$ to power the explosion and compared the calculated host metallicity versus Ni mass trend to the Timmes et al. (2003) model. Their data are consistent with the model, although with a higher scatter than predicted by the theory. They also found that varying the $\mathrm{O} / \mathrm{Fe}$ ratio according to thin or thick-disk models produced no substantial change in their results.

If the dependance of ${ }^{56} \mathrm{Ni}$ mass on host metallicity is real, then fainter and faster declining SNe Ia should be associated with higher metallicity, and thus more massive, host galaxies. If a decline in the rate of fast fading, fainter $\mathrm{SNe}$ Ia with redshift could be detected, it would be evidence for the relation between $\mathrm{Ni}$ mass and metallicity because we expect the more distant universe to consist of lower-mass and lower-metallicity objects on average when compared with the local universe. At this time, however, Malmquist biases complicate an accurate determination of the rate evolution for low-luminosity SNe Ia (Foley et al. 2008; S. Gonzalez-Gaitan et al. 2010, in preparation). We therefore look to host properties of lowluminosity $\mathrm{SNe}$ Ia in the local universe, where they are easier to detect and apparently more abundant. We use this larger sample to see if the fainter SNe do appear in higher-mass, highermetallicity galaxies on average.

To compare host properties with SN Ia variation, we construct a low-redshift sample of SNe Ia with well-observed light curves that provide a light-curve shape parameter, called stretch, the observed maximum light color, and the peak luminosity of the SN in the rest-frame $B$ band (Conley et al. 2008). We characterize the hosts of these $\mathrm{SNe}$ using newly generated and catalog galaxy integrated UV and optical magnitudes measured within matched apertures as inputs to an SED fitting program that estimates host stellar mass, luminosity-weighted age and star formation rate (SFR). We first use these results to explore the relation between the observed light-curve parameters and the derived host properties and compare our results to studies at higher redshift using the same methods (Sullivan et al. 2006; H09). We isolate a subset of hosts with low internal extinction in order to explore the relationship between SN peak brightness and host properties where the source of line-of-sight color for the $\mathrm{SNe}$ is minimized. We then extend the work of H09 by applying their techniques to our local sample to derive SN Ia ${ }^{56} \mathrm{Ni}$ mass, from the light-curve photometry, and compare these with host metallicities, from our derived host stellar masses, and host luminosity-weighted ages. Throughout this paper, we express host masses in $M_{\odot}$ and SFR in $M_{\odot} \mathrm{yr}^{-1}$, and assume a Hubble constant of $H_{0}=73 \mathrm{~km} \mathrm{~s}^{-1} \mathrm{Mpc}^{-1}$.

\section{SAMPLE SELECTION AND ANALYSIS}

Here we outline the properties of our low-redshift SN Ia sample and describe the methods we use to characterize the SN and host galaxy properties.

\subsection{The Local SN Sample and Light-curve Fitting}

For our local sample, we start with the low- $z$ literature sample summarized in Conley et al. (2008). We supplement this with more recent SN photometry from Hicken et al. (2009a). The nearby SNe Ia that we consider here are required to have modern CCD photometry with error bars, and are required to have phase coverage beyond 3 days after maximum light and prior to 7 days after maximum light. They are also required to have one restframe $B$-band magnitude between 8 days before and 13 days after maximum light and one rest-frame $U$ - or $V$-band magnitude in the same phase range. In addition, the wavelength range of the filters must be between 2700 and $7200 \AA$.

To be consistent with $\mathrm{H} 09$, we adopt the SN Ia light-curve fitting method from Conley et al. (2008). This method fits the SN light-curve data to produce the stretch, $s$ (Perlmutter et al. 1997), a peak rest-frame $B$-band apparent magnitude, $m_{B \max }$, and a SN color, $\mathcal{C}$, which is somewhat like $(B-V)_{B \max }$ in the sense that redder $\mathrm{SNe}$ have higher $\mathcal{C}$ values. Briefly, the stretch parameter is a time-axis scale factor that is applied to the light curve that aligns its shape with a "canonical" SN Ia light curve. The sense of the stretch parameter is such that faster, fainter $\mathrm{SNe}$ Ia have low-stretch values, and brighter, slower SNe Ia have higher-stretch values. The sense of the SN color parameter is such that bluer $\mathrm{SNe}$ at maximum are brighter. The corrected peak brightness of the SN Ia used for cosmology is determined by a linear combination of $s, \mathcal{C}$, and $m_{B \max }$ (Astier et al. 2006; Conley et al. 2008). Conley et al. (2008) present a much more detailed description of this method including comparisons with other popular fitting routines. These comparisons demonstrate that, at least for this study, our results are not dependant on the fitting technique.

Figure 1 shows distributions of the fitted SN light-curve properties for all the low-redshift SNe passing the previously described criteria in hosts with sufficient integrated host photometry to provide good SED fits (168 SNe Ia, filled histograms). This is the base sample we will examine in this work. The figure shows the distributions in stretch, peak color, absolute $B$ magnitude (uncorrected for stretch-color), and recession velocity. The light-curve fit properties of this sample are given in Table 1, which lists the SN name, the host galaxy, the recession velocity, the light-curve stretch, the fitted apparent $B$ magnitude at maximum light, and a status column which is described below. More details of the light-curve fits for these $\mathrm{SNe}$ can be found in A. Conley et al. (2010, in preparation).

Low-luminosity SNe Ia are generally better represented in lower-redshift samples for multiple reasons. The spectra and the stretch-luminosity and color-luminosity relations for lowstretch SNe Ia differ enough from higher-stretch $\mathrm{SNe}$ (Garnavich 
Table 1

SN Ia Sample

\begin{tabular}{|c|c|c|c|c|c|c|c|c|c|c|c|}
\hline SN & HOST & $\begin{array}{c}c z \\
\left.(\operatorname{log~km~s})^{-1}\right)\end{array}$ & Stretch $(\mathrm{s})^{\mathrm{a}}$ & $m_{B \max }$ & $\overline{\text { Status }^{\mathrm{b}}}$ & SN & HOST & $\begin{array}{c}c z \\
\left(\log \mathrm{km} \mathrm{s}^{-1}\right)\end{array}$ & Stretch $(\mathrm{s})^{\mathrm{a}}$ & $m_{B \max }$ & Status $^{\mathrm{b}}$ \\
\hline $1980 \mathrm{~N}$ & NGC 1316 & 3.26 & $0.83 \pm 0.01$ & $12.42 \pm 0.01$ & & $2001 \mathrm{~V}$ & NGC 3987 & 3.66 & $1.13 \pm 0.01$ & $14.62 \pm 0.02$ & $\mathrm{H}, \mathrm{C}$ \\
\hline 1981B & NGC 4536 & 3.26 & $0.88 \pm 0.02$ & $11.95 \pm 0.01$ & $\mathrm{C}$ & 2001ay & IC4423 & 3.96 & $1.59 \pm 0.03$ & $16.77 \pm 0.03$ & $\mathrm{H}$ \\
\hline 1981D & NGC 1316 & 3.26 & $0.86 \pm 0.04$ & $12.54 \pm 0.05$ & $\mathrm{C}$ & $2001 \mathrm{az}$ & UGC 10483 & 4.09 & $1.08 \pm 0.06$ & $16.93 \pm 0.04$ & $\mathrm{H}, \mathrm{C}$ \\
\hline $1990 \mathrm{~N}$ & NGC 4639 & 3.00 & $1.09 \pm 0.01$ & $12.70 \pm 0.02$ & $\mathrm{C}$ & $2001 \mathrm{ba}$ & M-05-28-01 & 3.95 & $1.01 \pm 0.02$ & $16.21 \pm 0.03$ & $\mathrm{H}, \mathrm{C}$ \\
\hline $1991 \mathrm{~T}$ & NGC 4527 & 3.24 & $1.07 \pm 0.02$ & $11.44 \pm 0.03$ & & $2001 \mathrm{da}$ & NGC 7780 & 3.71 & $0.89 \pm 0.24$ & $15.48 \pm 0.09$ & $\mathrm{H}, \mathrm{C}$ \\
\hline $1991 U$ & IC4232 & 3.98 & $1.01 \pm 0.05$ & $16.33 \pm 0.08$ & $\mathrm{H}, \mathrm{C}$ & $2001 \mathrm{el}$ & NGC 1448 & 3.07 & $0.98 \pm 0.01$ & $12.78 \pm 0.01$ & $\mathrm{C}$ \\
\hline 1991ag & IC4919 & 3.64 & $1.10 \pm 0.03$ & $14.43 \pm 0.08$ & $\mathrm{H}, \mathrm{C}$ & 2001en & NGC 523 & 3.69 & $0.90 \pm 0.05$ & $15.05 \pm 0.08$ & $\mathrm{H}, \mathrm{C}$ \\
\hline $1992 \mathrm{~A}$ & NGC 1380 & 3.27 & $0.81 \pm 0.01$ & $12.54 \pm 0.01$ & $\mathrm{C}$ & 2001ep & NGC 1699 & 3.59 & $0.88 \pm 0.02$ & $14.90 \pm 0.02$ & $\mathrm{C}$ \\
\hline $1992 \mathrm{P}$ & IC3690 & 3.88 & $1.16 \pm 0.12$ & $16.05 \pm 0.03$ & $\mathrm{H}, \mathrm{C}$ & $2001 \mathrm{fe}$ & UGC 5129 & 3.61 & $1.09 \pm 0.03$ & $14.70 \pm 0.02$ & $\mathrm{H}, \mathrm{C}$ \\
\hline 1992ag & E508-G67 & 3.88 & $0.98 \pm 0.05$ & $16.27 \pm 0.05$ & $\mathrm{H}, \mathrm{C}$ & 2001ie & UGC 5542 & 3.97 & $0.80 \pm 0.05$ & $16.78 \pm 0.05$ & $\mathrm{~L}, \mathrm{H}, \mathrm{C}$ \\
\hline $1992 b c$ & E300-G09 & 3.76 & $1.07 \pm 0.01$ & $15.10 \pm 0.01$ & $\mathrm{H}, \mathrm{C}$ & $2002 b f$ & CGCG266-031 & 3.86 & $0.94 \pm 0.03$ & $16.33 \pm 0.05$ & $\mathrm{H}, \mathrm{C}$ \\
\hline $1992 \mathrm{bl}$ & E291-G11 & 4.12 & $0.78 \pm 0.03$ & $17.31 \pm 0.05$ & $\mathrm{~L}, \mathrm{H}, \mathrm{C}$ & 2002bo & NGC 3190 & 3.11 & $0.94 \pm 0.01$ & $13.96 \pm 0.02$ & $\mathrm{C}$ \\
\hline 1992bo & E352-G57 & 3.75 & $0.75 \pm 0.01$ & $15.73 \pm 0.02$ & $\mathrm{~L}, \mathrm{H}, \mathrm{C}$ & $2002 \mathrm{~cd}$ & NGC 6916 & 3.49 & $1.07 \pm 0.02$ & $15.50 \pm 0.16$ & $\mathrm{C}$ \\
\hline $1993 \mathrm{H}$ & E445-G66 & 3.86 & $0.66 \pm 0.06$ & $16.79 \pm 0.03$ & $\mathrm{~L}, \mathrm{H}, \mathrm{C}$ & $2002 \mathrm{ck}$ & UGC 10030 & 3.97 & $1.06 \pm 0.06$ & $16.28 \pm 0.12$ & $\mathrm{H}, \mathrm{C}$ \\
\hline 1993ae & UGC 1071 & 3.76 & $0.77 \pm 0.03$ & $16.24 \pm 0.05$ & $\mathrm{~L}, \mathrm{H}$ & $2002 \mathrm{cr}$ & NGC 5468 & 3.45 & $0.94 \pm 0.02$ & $14.20 \pm 0.02$ & $\mathrm{C}$ \\
\hline $1994 \mathrm{M}$ & NGC 4493 & 3.84 & $0.80 \pm 0.03$ & $16.21 \pm 0.04$ & $\mathrm{H}, \mathrm{C}$ & $2002 \mathrm{de}$ & NGC 6104 & 3.92 & $1.06 \pm 0.07$ & $16.68 \pm 0.02$ & $\mathrm{H}, \mathrm{C}$ \\
\hline 1994Q & PGC0059076 & 3.95 & $1.07 \pm 0.07$ & $16.36 \pm 0.08$ & $\mathrm{H}$ & $2002 \mathrm{dj}$ & NGC 5018 & 3.45 & $0.94 \pm 0.01$ & $13.93 \pm 0.04$ & $\mathrm{C}$ \\
\hline $1994 \mathrm{~S}$ & NGC 4495 & 3.66 & $1.04 \pm 0.04$ & $14.78 \pm 0.02$ & $\mathrm{H}, \mathrm{C}$ & $2002 d p$ & NGC 7678 & 3.54 & $0.96 \pm 0.04$ & $14.61 \pm 0.03$ & $\mathrm{C}$ \\
\hline 1994ae & NGC 3370 & 3.11 & $1.05 \pm 0.01$ & $12.95 \pm 0.02$ & $\mathrm{C}$ & $2002 \mathrm{er}$ & UGC 10743 & 3.43 & $0.88 \pm 0.01$ & $14.26 \pm 0.06$ & $\mathrm{C}$ \\
\hline $1995 \mathrm{D}$ & NGC 2962 & 3.29 & $1.11 \pm 0.01$ & $13.26 \pm 0.03$ & $\mathrm{C}$ & 2002es & UGC 2708 & 3.73 & $1.01 \pm 0.05$ & $16.16 \pm 0.10$ & $\mathrm{H}$ \\
\hline $1995 \mathrm{E}$ & NGC 2441 & 3.54 & $0.93 \pm 0.03$ & $16.69 \pm 0.02$ & $\mathrm{C}$ & $2002 \mathrm{fk}$ & NGC 1309 & 3.35 & $0.99 \pm 0.03$ & $13.13 \pm 0.03$ & $\mathrm{C}$ \\
\hline 1995al & NGC 3021 & 3.19 & $1.08 \pm 0.03$ & $13.32 \pm 0.02$ & $\mathrm{C}$ & $2002 \mathrm{ha}$ & NGC 6962 & 3.63 & $0.87 \pm 0.03$ & $14.71 \pm 0.05$ & $\mathrm{H}, \mathrm{C}$ \\
\hline 1995bd & UGC 3151 & 3.66 & $1.03 \pm 0.01$ & $15.28 \pm 0.20$ & $\mathrm{H}, \mathrm{C}$ & $2002 \mathrm{he}$ & UGC 4322 & 3.87 & $0.80 \pm 0.02$ & $16.23 \pm 0.04$ & $\mathrm{H}, \mathrm{C}$ \\
\hline $1996 \mathrm{C}$ & $M+08-25-47$ & 3.91 & $1.07 \pm 0.04$ & $16.63 \pm 0.03$ & $\mathrm{H}, \mathrm{C}$ & $2002 \mathrm{hu}$ & $M+06-06-12$ & 4.04 & $1.04 \pm 0.02$ & $16.63 \pm 0.02$ & $\mathrm{H}, \mathrm{C}$ \\
\hline $1996 \mathrm{X}$ & NGC 5061 & 3.31 & $0.85 \pm 0.02$ & $12.99 \pm 0.03$ & $\mathrm{C}$ & $2002 \mathrm{hw}$ & UGC 52 & 3.72 & $0.76 \pm 0.03$ & $16.65 \pm 0.05$ & $\mathrm{~L}, \mathrm{H}$ \\
\hline $1996 Z$ & NGC 2935 & 3.36 & $0.92 \pm 0.08$ & $14.32 \pm 0.08$ & $\mathrm{C}$ & 2002jy & NGC 477 & 3.77 & $1.12 \pm 0.04$ & $15.74 \pm 0.03$ & $\mathrm{H}, \mathrm{C}$ \\
\hline 1996ai & NGC 5005 & 3.00 & $1.11 \pm 0.03$ & $16.90 \pm 0.01$ & $\mathrm{R}, \mathrm{C}$ & $2003 \mathrm{U}$ & NGC 6365A & 3.93 & $0.79 \pm 0.02$ & $16.51 \pm 0.03$ & $\mathrm{~L}, \mathrm{H}, \mathrm{C}$ \\
\hline 1996bo & NGC 673 & 3.72 & $0.87 \pm 0.01$ & $15.84 \pm 0.03$ & $\mathrm{H}, \mathrm{C}$ & $2003 \mathrm{~W}$ & UGC 5234 & 3.78 & $0.99 \pm 0.02$ & $15.88 \pm 0.02$ & $\mathrm{H}, \mathrm{C}$ \\
\hline $1996 \mathrm{bv}$ & UGC 3432 & 3.70 & $1.05 \pm 0.05$ & $15.32 \pm 0.05$ & $\mathrm{H}, \mathrm{C}$ & $2003 \mathrm{cg}$ & NGC 3169 & 3.09 & $0.95 \pm 0.01$ & $15.79 \pm 0.02$ & $\mathrm{R}, \mathrm{C}$ \\
\hline $1997 \mathrm{E}$ & NGC 2258 & 3.60 & $0.81 \pm 0.02$ & $15.11 \pm 0.05$ & $\mathrm{C}$ & $2003 \mathrm{du}$ & UGC 9391 & 3.28 & $1.01 \pm 0.01$ & $13.47 \pm 0.01$ & $\mathrm{C}$ \\
\hline $1997 \mathrm{Y}$ & NGC 4675 & 3.68 & $0.91 \pm 0.07$ & $15.28 \pm 0.07$ & $\mathrm{H}$ & $2003 \mathrm{fa}$ & $\mathrm{M}+07-36-33$ & 4.07 & $1.15 \pm 0.01$ & $16.71 \pm 0.02$ & $\mathrm{H}, \mathrm{C}$ \\
\hline $1997 b p$ & NGC 4680 & 3.40 & $0.97 \pm 0.02$ & $13.91 \pm 0.02$ & $\mathrm{C}$ & 2003hu & A191131+7753 & 4.35 & $1.16 \pm 0.06$ & $18.46 \pm 0.14$ & $\mathrm{H}, \mathrm{C}$ \\
\hline $1997 \mathrm{bq}$ & NGC 3147 & 3.45 & $0.88 \pm 0.02$ & $14.33 \pm 0.04$ & & $2003 h x$ & NGC 2076 & 3.33 & $0.84 \pm 0.06$ & $14.86 \pm 0.05$ & $\mathrm{C}$ \\
\hline $1997 \mathrm{br}$ & E576-G40 & 3.32 & $0.91 \pm 0.04$ & $13.31 \pm 0.08$ & & $2003 i c$ & M-02-02-86 & 4.22 & $0.75 \pm 0.05$ & $17.66 \pm 0.08$ & $\mathrm{~L}, \mathrm{H}$ \\
\hline $1997 \mathrm{cw}$ & NGC 105 & 3.72 & $1.13 \pm 0.04$ & $15.99 \pm 0.06$ & $\mathrm{H}, \mathrm{C}$ & $2003 \mathrm{kc}$ & $M+05-23-37$ & 4.00 & $0.84 \pm 0.04$ & $17.14 \pm 0.05$ & $\mathrm{H}$ \\
\hline 1997do & UGC 3845 & 3.48 & $0.94 \pm 0.03$ & $14.27 \pm 0.04$ & $\mathrm{C}$ & $2003 \mathrm{kf}$ & M-02-16-02 & 3.35 & $1.04 \pm 0.03$ & $13.27 \pm 0.13$ & $\mathrm{C}$ \\
\hline $1998 \mathrm{~V}$ & NGC 6627 & 3.72 & $1.00 \pm 0.04$ & $15.08 \pm 0.08$ & $\mathrm{H}, \mathrm{C}$ & $2004 \mathrm{~L}$ & $M+03-27-38$ & 3.99 & $0.93 \pm 0.04$ & $17.30 \pm 0.05$ & $\mathrm{H}, \mathrm{C}$ \\
\hline $1998 \mathrm{ab}$ & NGC 4704 & 3.91 & $0.94 \pm 0.02$ & $16.06 \pm 0.03$ & $\mathrm{H}, \mathrm{C}$ & 2004as & A112539+2249 & 3.97 & $1.06 \pm 0.04$ & $16.96 \pm 0.02$ & $\mathrm{H}, \mathrm{C}$ \\
\hline $1998 \mathrm{aq}$ & NGC 3982 & 2.97 & $0.95 \pm 0.01$ & $12.31 \pm 0.01$ & $\mathrm{C}$ & $2004 \mathrm{eo}$ & NGC 6928 & 3.67 & $0.87 \pm 0.00$ & $15.08 \pm 0.05$ & $\mathrm{H}, \mathrm{C}$ \\
\hline 1998bu & NGC 3368 & 2.97 & $0.95 \pm 0.02$ & $12.10 \pm 0.01$ & $\mathrm{C}$ & $2004 \mathrm{fu}$ & NGC 6949 & 3.44 & $0.87 \pm 0.01$ & $14.25 \pm 0.16$ & $\mathrm{C}$ \\
\hline 1998de & NGC 252 & 3.70 & $0.57 \pm 0.02$ & $17.36 \pm 0.03$ & $\mathrm{~L}, \mathrm{H}, \mathrm{C}$ & $2005 \mathrm{am}$ & NGC 2811 & 3.40 & $0.70 \pm 0.05$ & $13.66 \pm 0.03$ & $\mathrm{~L}, \mathrm{C}$ \\
\hline $1998 \mathrm{dh}$ & NGC 7541 & 3.43 & $0.91 \pm 0.02$ & $13.86 \pm 0.04$ & & $2005 \mathrm{eq}$ & M-01-09-06 & 3.95 & $1.18 \pm 0.01$ & $16.30 \pm 0.03$ & $\mathrm{H}, \mathrm{C}$ \\
\hline $1998 \mathrm{dm}$ & M-01-04-44 & 3.29 & $1.07 \pm 0.07$ & $14.64 \pm 0.08$ & $\mathrm{C}$ & $2005 \mathrm{hc}$ & $\mathrm{M}+00-06-03$ & 4.14 & $1.02 \pm 0.03$ & $17.36 \pm 0.02$ & $\mathrm{H}, \mathrm{C}$ \\
\hline $1998 \mathrm{dx}$ & UGC 11149 & 4.21 & $0.80 \pm 0.04$ & $17.55 \pm 0.04$ & $\mathrm{~L}, \mathrm{H}, \mathrm{C}$ & $2005 \mathrm{hk}$ & UGC 272 & 3.59 & $0.88 \pm 0.00$ & $15.95 \pm 0.01$ & \\
\hline $1998 \mathrm{ec}$ & UGC 3576 & 3.77 & $0.98 \pm 0.07$ & $16.17 \pm 0.11$ & $\mathrm{H}$ & $2005 \mathrm{iq}$ & M-03-01-08 & 4.01 & $0.87 \pm 0.02$ & $16.82 \pm 0.02$ & $\mathrm{H}, \mathrm{C}$ \\
\hline $1998 \mathrm{eg}$ & $\mathrm{M}+01-57-14$ & 3.87 & $0.92 \pm 0.06$ & $16.11 \pm 0.05$ & $\mathrm{H}$ & $2005 \mathrm{ir}$ & A011643+0047 & 4.36 & $1.44 \pm 0.11$ & $18.42 \pm 0.03$ & $\mathrm{H}, \mathrm{C}$ \\
\hline $1998 \mathrm{es}$ & NGC 632 & 3.50 & $1.14 \pm 0.01$ & $13.84 \pm 0.02$ & $\mathrm{C}$ & $2005 \mathrm{kc}$ & NGC 7311 & 3.65 & $0.93 \pm 0.02$ & $15.61 \pm 0.06$ & $\mathrm{H}, \mathrm{C}$ \\
\hline 1999X & CGCG180-22 & 3.88 & $0.91 \pm 0.08$ & $16.09 \pm 0.13$ & $\mathrm{H}, \mathrm{C}$ & $2005 \mathrm{ke}$ & NGC 1371 & 3.16 & $0.64 \pm 0.04$ & $14.80 \pm 0.03$ & $\mathrm{~L}$ \\
\hline 1999aa & NGC 2595 & 3.65 & $1.13 \pm 0.01$ & $14.73 \pm 0.02$ & $\mathrm{H}, \mathrm{C}$ & $2005 \mathrm{ki}$ & NGC 3332 & 3.76 & $0.80 \pm 0.01$ & $15.55 \pm 0.03$ & $\mathrm{H}, \mathrm{C}$ \\
\hline $1999 \mathrm{ac}$ & NGC 6063 & 3.45 & $0.98 \pm 0.01$ & $14.12 \pm 0.02$ & $\mathrm{C}$ & 20051s & $\mathrm{M}+07-07-01$ & 3.80 & $1.13 \pm 0.03$ & $16.25 \pm 0.04$ & $\mathrm{H}, \mathrm{C}$ \\
\hline $1999 \mathrm{cc}$ & NGC 6038 & 3.97 & $0.78 \pm 0.02$ & $16.78 \pm 0.01$ & $\mathrm{~L}, \mathrm{H}, \mathrm{C}$ & $2005 \mathrm{mc}$ & UGC 4414 & 3.88 & $0.65 \pm 0.04$ & $17.23 \pm 0.02$ & $\mathrm{~L}, \mathrm{H}$ \\
\hline $1999 \mathrm{cl}$ & NGC 4501 & 3.33 & $0.96 \pm 0.02$ & $14.87 \pm 0.02$ & $\mathrm{R}, \mathrm{C}$ & $2005 \mathrm{~ms}$ & UGC 4614 & 3.88 & $1.06 \pm 0.02$ & $16.16 \pm 0.02$ & $\mathrm{H}, \mathrm{C}$ \\
\hline $1999 \mathrm{cp}$ & NGC 5468 & 3.45 & $1.01 \pm 0.03$ & $13.95 \pm 0.02$ & $\mathrm{C}$ & $2005 \mathrm{mz}$ & NGC 1275 & 3.72 & $0.61 \pm 0.02$ & $16.42 \pm 0.07$ & $\mathrm{~L}, \mathrm{H}$ \\
\hline 1999da & NGC 6411 & 3.57 & $0.55 \pm 0.02$ & $16.60 \pm 0.03$ & $\mathrm{~L}, \mathrm{C}$ & $2006 \mathrm{~N}$ & $M+11-08-12$ & 3.63 & $0.76 \pm 0.02$ & $15.08 \pm 0.04$ & $\mathrm{~L}, \mathrm{H}, \mathrm{C}$ \\
\hline $1999 \mathrm{dk}$ & UGC 1087 & 3.66 & $0.97 \pm 0.03$ & $14.83 \pm 0.03$ & $\mathrm{H}, \mathrm{C}$ & $2006 \mathrm{~S}$ & UGC 7934 & 3.98 & $1.12 \pm 0.01$ & $16.86 \pm 0.01$ & $\mathrm{H}, \mathrm{C}$ \\
\hline $1999 \mathrm{dq}$ & NGC 976 & 3.64 & $1.12 \pm 0.01$ & $14.42 \pm 0.05$ & $\mathrm{H}, \mathrm{C}$ & $2006 X$ & NGC 4321 & 3.20 & $0.96 \pm 0.01$ & $15.22 \pm 0.01$ & $\mathrm{R}, \mathrm{C}$ \\
\hline 1999ee & IC5179 & 3.53 & $1.07 \pm 0.01$ & $14.85 \pm 0.01$ & $\mathrm{C}$ & $2006 \mathrm{ac}$ & NGC 4619 & 3.83 & $0.87 \pm 0.02$ & $16.18 \pm 0.02$ & $\mathrm{H}, \mathrm{C}$ \\
\hline $1999 \mathrm{gd}$ & NGC 2623 & 3.74 & $0.92 \pm 0.06$ & $16.93 \pm 0.03$ & $\mathrm{H}, \mathrm{C}$ & 2006ak & A110932+2837 & 4.05 & $0.84 \pm 0.04$ & $17.24 \pm 0.10$ & $\mathrm{H}, \mathrm{C}$ \\
\hline $2000 \mathrm{E}$ & NGC 6951 & 3.12 & $1.06 \pm 0.01$ & $12.85 \pm 0.15$ & $\mathrm{C}$ & 2006al & A103929+0511 & 4.31 & $0.78 \pm 0.04$ & $18.44 \pm 0.05$ & $\mathrm{~L}, \mathrm{H}, \mathrm{C}$ \\
\hline $2000 \mathrm{ca}$ & E383-G32 & 3.86 & $1.08 \pm 0.03$ & $15.57 \pm 0.03$ & $\mathrm{H}, \mathrm{C}$ & 2006ar & $M+11-13-36$ & 3.83 & $0.92 \pm 0.03$ & $16.48 \pm 0.01$ & $\mathrm{H}, \mathrm{C}$ \\
\hline 2000ce & UGC 4195 & 3.69 & $1.02 \pm 0.03$ & $17.06 \pm 0.04$ & $\mathrm{H}, \mathrm{C}$ & $2006 a x$ & NGC 3663 & 3.70 & $1.00 \pm 0.01$ & $15.04 \pm 0.02$ & $\mathrm{H}, \mathrm{C}$ \\
\hline $2000 \mathrm{cx}$ & NGC 524 & 3.38 & $0.87 \pm 0.01$ & $13.06 \pm 0.04$ & & $2006 a z$ & NGC 4172 & 3.97 & $0.86 \pm 0.01$ & $16.49 \pm 0.01$ & $\mathrm{H}, \mathrm{C}$ \\
\hline $2000 \mathrm{dk}$ & NGC 382 & 3.72 & $0.74 \pm 0.01$ & $15.36 \pm 0.03$ & $\mathrm{~L}, \mathrm{H}, \mathrm{C}$ & $2006 \mathrm{bk}$ & $\mathrm{M}+06-33-20$ & 4.17 & $1.10 \pm 0.03$ & $17.00 \pm 0.06$ & $\mathrm{H}$ \\
\hline $2000 \mathrm{fa}$ & UGC 3770 & 3.80 & $1.03 \pm 0.03$ & $15.86 \pm 0.05$ & $\mathrm{H}, \mathrm{C}$ & $2006 \mathrm{bq}$ & NGC 6685 & 3.82 & $0.84 \pm 0.02$ & $16.15 \pm 0.04$ & $\mathrm{H}, \mathrm{C}$ \\
\hline $2001 N$ & NGC 3327 & 3.80 & $0.94 \pm 0.05$ & $16.58 \pm 0.04$ & $\mathrm{H}, \mathrm{C}$ & $2006 \mathrm{br}$ & NGC 5185 & 3.87 & $0.81 \pm 0.04$ & $18.95 \pm 0.03$ & $\mathrm{R}, \mathrm{H}, \mathrm{C}$ \\
\hline
\end{tabular}

Table 1

(Continued) 
Table 1

(Continued)

\begin{tabular}{|c|c|c|c|c|c|}
\hline SN & HOST & $\begin{array}{c}c z \\
\left(\log \mathrm{km} \mathrm{s}^{-1}\right)\end{array}$ & Stretch $(\mathrm{s})^{\mathrm{a}}$ & $m_{B \max }$ & Status $^{b}$ \\
\hline $2006 b t$ & $M+03-41-04$ & 3.98 & $1.01 \pm 0.02$ & $16.95 \pm 0.02$ & $\mathrm{H}, \mathrm{C}$ \\
\hline 2006bw & A143356+0347 & 4.00 & $0.72 \pm 0.03$ & $17.56 \pm 0.04$ & $\mathrm{~L}, \mathrm{H}, \mathrm{C}$ \\
\hline $2006 \mathrm{cc}$ & UGC 10244 & 3.99 & $1.03 \pm 0.01$ & $17.81 \pm 0.01$ & $\mathrm{H}, \mathrm{C}$ \\
\hline $2006 \mathrm{cj}$ & A125924+2820 & 4.31 & $1.25 \pm 0.11$ & $18.14 \pm 0.03$ & $\mathrm{H}, \mathrm{C}$ \\
\hline $2006 \mathrm{~cm}$ & UGC 11723 & 3.69 & $1.05 \pm 0.04$ & $17.94 \pm 0.03$ & $\mathrm{R}, \mathrm{H}, \mathrm{C}$ \\
\hline $2006 \mathrm{cp}$ & UGC 7357 & 3.82 & $1.07 \pm 0.02$ & $15.99 \pm 0.02$ & $\mathrm{H}, \mathrm{C}$ \\
\hline 2006ef & NGC 809 & 3.73 & $0.84 \pm 0.04$ & $15.16 \pm 0.17$ & $\mathrm{H}$ \\
\hline 2006еј & NGC 191A & 3.79 & $0.81 \pm 0.04$ & $15.79 \pm 0.03$ & $\mathrm{H}, \mathrm{C}$ \\
\hline 2006en & $M+05-54-41$ & 3.98 & $1.00 \pm 0.04$ & $16.75 \pm 0.05$ & $\mathrm{H}, \mathrm{C}$ \\
\hline $2006 \mathrm{gj}$ & UGC 2650 & 3.93 & $0.65 \pm 0.07$ & $17.61 \pm 0.05$ & $\mathrm{~L}, \mathrm{H}, \mathrm{C}$ \\
\hline $2006 \mathrm{gz}$ & IC1277 & 3.85 & $1.26 \pm 0.01$ & $15.84 \pm 0.03$ & $\mathrm{H}$ \\
\hline 2006hb & M-04-12-34 & 3.66 & $0.67 \pm 0.06$ & $15.48 \pm 0.05$ & $\mathrm{~L}, \mathrm{H}, \mathrm{C}$ \\
\hline $2006 \mathrm{kf}$ & UGC 2829 & 3.80 & $0.71 \pm 0.03$ & $15.83 \pm 0.10$ & $\mathrm{~L}, \mathrm{H}, \mathrm{C}$ \\
\hline 2006le & UGC 3218 & 3.72 & $1.11 \pm 0.01$ & $14.80 \pm 0.17$ & $\mathrm{H}, \mathrm{C}$ \\
\hline $2006 \mathrm{mo}$ & $M+06-02-17$ & 4.05 & $0.75 \pm 0.03$ & $17.44 \pm 0.03$ & $\mathrm{~L}, \mathrm{H}, \mathrm{C}$ \\
\hline $2006 n z$ & A005629-0113 & 4.06 & $0.60 \pm 0.10$ & $18.08 \pm 0.04$ & $\mathrm{~L}, \mathrm{H}$ \\
\hline 2006 ob & UGC 1333 & 4.25 & $0.71 \pm 0.01$ & $18.23 \pm 0.02$ & $\mathrm{~L}, \mathrm{H}, \mathrm{C}$ \\
\hline 2006on & A215558-0104 & 4.32 & $0.99 \pm 0.09$ & $18.41 \pm 0.07$ & $\mathrm{H}, \mathrm{C}$ \\
\hline 2006 os & UGC 2384 & 3.99 & $0.91 \pm 0.03$ & $17.61 \pm 0.06$ & $\mathrm{H}$ \\
\hline $2006 \mathrm{sr}$ & UGC 14 & 3.86 & $0.84 \pm 0.02$ & $16.13 \pm 0.04$ & $\mathrm{H}, \mathrm{C}$ \\
\hline 2006te & A081144+4133 & 3.98 & $1.03 \pm 0.05$ & $16.51 \pm 0.04$ & $\mathrm{H}, \mathrm{C}$ \\
\hline $2007 \mathrm{~F}$ & UGC 8162 & 3.85 & $1.07 \pm 0.01$ & $15.90 \pm 0.01$ & $\mathrm{H}, \mathrm{C}$ \\
\hline $2007 \mathrm{O}$ & UGC 9612 & 4.03 & $0.93 \pm 0.04$ & $16.78 \pm 0.05$ & $\mathrm{H}, \mathrm{C}$ \\
\hline 2007R & UGC 4008 & 3.96 & $0.82 \pm 0.02$ & $16.65 \pm 0.05$ & $\mathrm{H}$ \\
\hline $2007 \mathrm{~S}$ & UGC 5378 & 3.62 & $1.13 \pm 0.01$ & $15.82 \pm 0.01$ & $\mathrm{H}, \mathrm{C}$ \\
\hline $2007 \mathrm{ae}$ & UGC 10704 & 4.29 & $1.15 \pm 0.03$ & $17.78 \pm 0.04$ & $\mathrm{H}, \mathrm{C}$ \\
\hline 2007af & NGC 5584 & 3.20 & $0.96 \pm 0.01$ & $13.16 \pm 0.02$ & $\mathrm{C}$ \\
\hline 2007ap & $M+03-41-03$ & 3.67 & $0.54 \pm 0.07$ & $15.86 \pm 0.03$ & $\mathrm{~L}, \mathrm{H}, \mathrm{C}$ \\
\hline 2007au & UGC 3725 & 3.79 & $0.66 \pm 0.03$ & $16.51 \pm 0.03$ & $\mathrm{~L}, \mathrm{H}, \mathrm{C}$ \\
\hline $2007 b c$ & UGC 6332 & 3.80 & $0.84 \pm 0.03$ & $15.89 \pm 0.02$ & $\mathrm{H}, \mathrm{C}$ \\
\hline $2007 \mathrm{bd}$ & UGC 4455 & 3.97 & $0.82 \pm 0.01$ & $16.57 \pm 0.02$ & $\mathrm{H}, \mathrm{C}$ \\
\hline $2007 \mathrm{bm}$ & NGC 3672 & 3.27 & $0.92 \pm 0.01$ & $14.48 \pm 0.02$ & $\mathrm{C}$ \\
\hline $2007 \mathrm{bz}$ & IC3918 & 3.81 & $1.17 \pm 0.03$ & $16.67 \pm 0.03$ & $\mathrm{H}, \mathrm{C}$ \\
\hline $2007 \mathrm{cg}$ & E508-G75 & 4.00 & $0.82 \pm 0.05$ & $18.28 \pm 0.07$ & $\mathrm{H}, \mathrm{C}$ \\
\hline $2007 \mathrm{ci}$ & NGC 3873 & 3.74 & $0.75 \pm 0.01$ & $15.92 \pm 0.02$ & $\mathrm{~L}, \mathrm{H}, \mathrm{C}$ \\
\hline $2007 \mathrm{sr}$ & NGC 4038 & 3.21 & $0.99 \pm 0.02$ & $12.76 \pm 0.04$ & $\mathrm{C}$ \\
\hline 2008af & UGC 9640 & 4.00 & $0.85 \pm 0.03$ & $16.78 \pm 0.08$ & $\mathrm{H}, \mathrm{C}$ \\
\hline $2008 b f$ & NGC 4055 & 3.86 & $1.04 \pm 0.02$ & $15.73 \pm 0.02$ & $\mathrm{H}, \mathrm{C}$ \\
\hline
\end{tabular}

Notes.

a Stretch errors for SNe with $s<0.7$ have been multiplied by 3.0.

${ }^{b} \mathrm{~L}$ : low stretch, $s<0.80, \mathrm{R}$ : red, $\mathcal{C}>0.7, \mathrm{H}$ : in Hubble flow, $z>0.0133, \mathrm{C}$ : eligible for cosmology fitting.

et al. 2004; Taubenberger et al. 2008) to increase the errors in fitting their light curves beyond the error-budget requirement for cosmological parameter determination. This, combined with their faintness, makes low-stretch SNe less appealing targets for the spectroscopic follow-up required for cosmological surveys. As a result, the SN Ia training set derived for cosmology in Conley et al. (2008) contains no SNe with $s<0.70$, and very few below $s=0.75$. At lower redshifts where even faint $\mathrm{SNe}$ are relatively easy to follow up, the unusual objects tend to attract attention and acquire spectroscopy.

The break in the stretch-luminosity relation appears to be at $s \sim 0.80$ (S. Gonzalez-Gaitan et al. 2010, in preparation). For this paper, we define the set of low-luminosity $\mathrm{SNe}$ Ia based on stretch to be those with $s<0.80$ (to the left of the dashed vertical line in Figure 1(a)). The training set from Conley et al. (2008) allows us to extend this limit slightly to $s=0.75$ when we apply corrections for stretch-luminosity.

Our local sample contains 29 SNe with $s<0.80$ of which 12 are below $s=0.70$. Since we are using a light-curve method
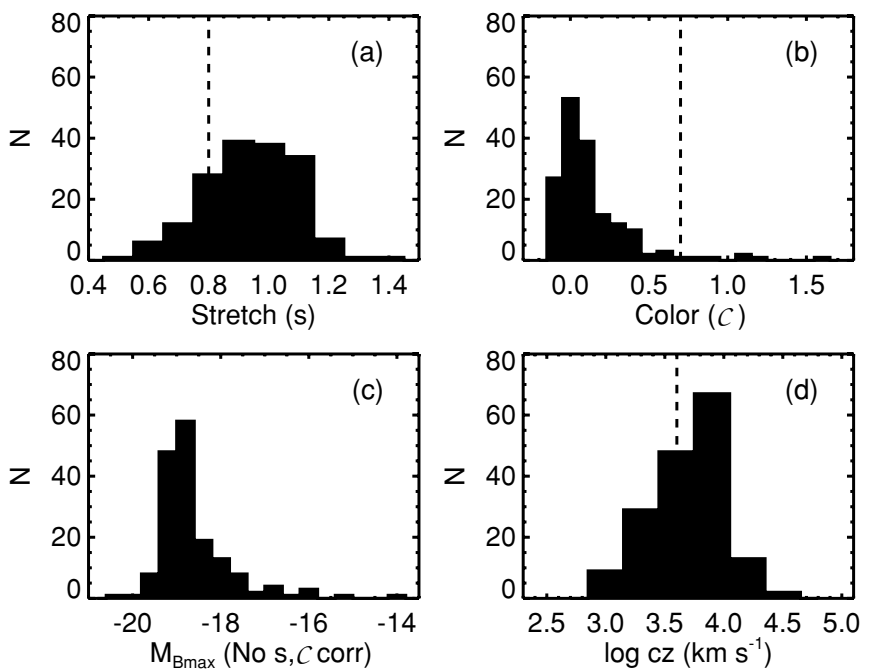

Figure 1. Frequency diagrams of light-curve fit parameters for the local SNe Ia having enough observations to determine a stretch value and having sufficient host photometry to allow good fits to their SED: (a) stretch, (b) color, (c) $M_{B \max }$ (uncorrected for stretch color), and (d) recession velocity in $\mathrm{km} \mathrm{s}^{-1}$. The vertical dashed lines show various limits described in the text: (a) the limit between ordinary and low-luminosity $\mathrm{SNe} \mathrm{Ia}(s=0.80)$, (b) the peak color limit above which the fitting becomes less accurate $(\mathcal{C}=0.7)$, and $(d)$ the Hubble flow limit adopted here $\left(c z=4000 \mathrm{~km} \mathrm{~s}^{-1}, z=0.013\right)$. There are $168 \mathrm{SNe} \mathrm{Ia}$ in the sample with well-fitted host SEDs.

developed for cosmology, this means our fitting can only tell that an object with $s<0.70$ is low stretch, but cannot accurately measure the stretch. We have increased the error bars by a factor of 3 for objects with $s<0.70$. The sample used in H09 was derived from the Supernova Legacy Survey (SNLS; Astier et al. 2006), which was optimized for cosmology. Either due to the fact that such objects are very rare beyond $z=0.2$, or because the spectroscopic follow-up was biased against them or both, there are no objects with $s<0.75$ in the H09 sample (see also Bronder et al. 2008). The low-stretch SNe Ia in our sample are indicated with an " $L$ " in the status column in Table 1.

A similar situation exists for the peak color, $\mathcal{C}$. SNe Ia with colors greater than $\mathcal{C} \sim 0.7$ (see Figure $1(\mathrm{~b})$ ) are also unappealing objects for cosmological surveys because they are fainter and because they have larger fitting errors. These red SNe Ia should not be excluded when examining host properties, however. Our sample contains six $\mathrm{SNe}$ Ia with $\mathcal{C}>0.7$. These $\mathrm{SNe}$ are indicated with an " $\mathrm{R}$ " in the status column of Table 1. We have increased the peak color errors by a factor of 10 for these $\mathrm{SNe}$ to reflect the higher uncertainty in this parameter.

We emphasize that while the local sample assembled here is appropriate for exploring the link between host and SN properties, many of the $\mathrm{SNe}$ in our sample are too local $\left(c z<4000 \mathrm{~km} \mathrm{~s}^{-1}, z<0.013\right.$, to the left of the vertical dashed line in Figure 1(d)) or have stretch errors or colors large enough to exclude them from use in cosmological fits. We indicate the cosmology status of our sample SNe Ia in the last column of Table 1 where an $\mathrm{H}$ indicates a SN Ia in the Hubble flow $\left(c z>4000 \mathrm{~km} \mathrm{~s}^{-1}\right)$, and a $\mathrm{C}$ indicates a $\mathrm{SN}$ Ia that is eligible for cosmological fitting based on the light-curve fit quality and having parameters within the range where the light-curve fitting has reasonably low errors.

\subsection{Integrated Host Photometry and SED Fitting}

We assembled host integrated photometry in the ultraviolet (UV) and optical to characterize the SN Ia host SEDs. For our 
low-redshift host galaxy sample, the addition of the Galaxy Evolution Explorer (GALEX) UV photometry improves the estimation of short-term star formation (e.g., Martin et al. 2005, Figure 1), and provides similar constraints on recent star formation when compared with the higher-redshift samples from the SNLS, whose blue optical photometry corresponds to the rest-frame UV. All host magnitudes are total magnitudes using matched elliptical apertures having the standard D25 diameter, to be compatible with the RC3. All host magnitudes are corrected for Milky Way (foreground) extinction using the dust maps of Schlegel et al. (1998).

In the UV, we generated host integrated magnitudes as part of the preliminary efforts to produce the GALEX Large Galaxy Atlas (GLGA; M. Seibert et al. 2010, in preparation). This atlas will measure $\sim 20,000$ galaxies imaged by the GALEX UV-imaging satellite (Martin et al. 2005) having diameters in the UV greater than 1 arcmin. The GALEX imaging mode has two bandpasses, one in the far-UV (FUV; $\lambda_{\text {eff }}=1539 \AA$, $\Delta \lambda=442 \AA$ ) and another in the near-UV (NUV; $\lambda_{\text {eff }}=2316 \AA$, $\Delta \lambda=1060 \AA$ ). We use a technique similar to that used to generate the Nearby Galaxy Atlas (Gil de Paz et al. 2007) with which we cross-checked our integrated UV magnitudes. This method will be described in detail in M. Seibert et al. (2010, in preparation), but to summarize, we perform surface photometry in elliptical apertures on sky-subtracted images that have had foreground point sources and background galaxies masked.

For optical photometry, we used the RC3 (de Vaucouleurs et al. 1991) and/or images obtained from the Sloan Digital Sky Survey (SDSS $;{ }^{15}$ York et al. 2000) in the five SDSS bands: $u, g$, $r, i$, and $z$. Our sample required either SDSS coverage or an integrated magnitude from the RC3. The RC3 total integrated Johnson ultraviolet, blue, visual (UBV) magnitudes were obtained directly from NASA/IPAC Extragalactic Database (NED). ${ }^{16}$ For the larger hosts in our sample, we found the SDSS catalog data to be inaccurate for a number of reasons. Some of these hosts spanned multiple image strips and many were broken up into sub-regions making the determination of a total flux problematic. In order to enforce consistency across wavelengths, we decided to coadd and mosaic the SDSS image data for each SN host and derive the integrated photometry ourselves. To achieve this, we adapted our integrated photometry methods developed for the GLGA to SDSS image data. The major difference between GALEX and SDSS data is in the treatment of the sky background, which is extremely low in GALEX data. We checked the consistency of our integrated SDSS magnitudes by comparing them with RC3 magnitudes and found them to agree within the errors. All UV and optical integrated galaxy magnitudes are presented in M. Seibert et al. (2010, in preparation).

Sullivan et al. (2006) fit optical photometry to galaxy SED models produced with the PEGASE.2 SED galaxy spectral evolution code (Fioc \& Rocca-Volmerange 1997; Le Borgne \& Rocca-Volmerange 2002; Le Borgne et al. 2004). We adopted this method to allow a direct comparison with the higher-redshift host studies of Sullivan et al. (2006) and H09. As with these studies, all redshifts are known from the hosted SNe. This has the benefit of eliminating redshift degeneracies in the SED fits. The average internal extinction was allowed to vary in the fits over the range $0.0<E(B-V)_{\text {HOST }}<0.7$ in increments of 0.05 mag using a Calzetti et al. (1994) dust model (see Fioc \& Rocca-Volmerange 1997). We point out that the stellar mass derived from PEGASE. 2 models is an estimate of the current

\footnotetext{
15 http://www.sdss.org

16 http://nedwww.ipac.caltech.edu/
}

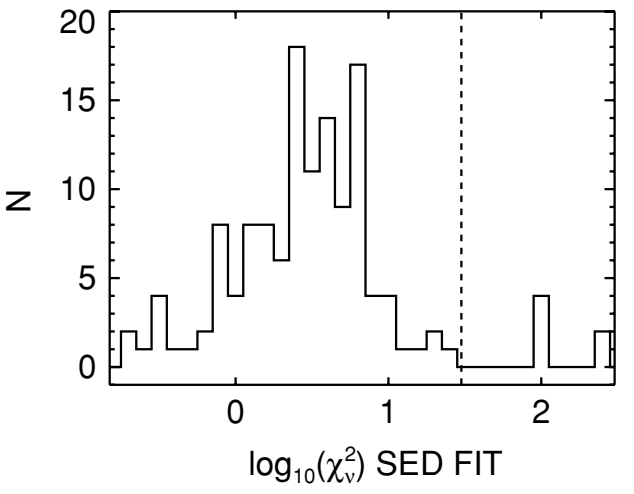

Figure 2. Frequency diagram of $\chi_{v}^{2}$ of the host SED fits illustrating one criterion for eliminating unusable hosts: we require $\chi_{v}^{2}<30$ for the fit. Large values of $\chi_{v}^{2}$ arise because local hosts have smaller photometric errors than hosts at higher redshifts. The other criteria require a unique solution for the SED and require UV and optical photometric points in each SED. These criteria reduce the sample of SNe Ia with good light curves from 258 down to 168 and produce 166 unique host SED fits (two galaxies in our sample hosted two SNe Ia each; see the text).

mass in stars and is not the integral of the star formation history for a given galaxy (Sullivan et al. 2006). We also emphasize that our ages are strongly influenced by the flux produced by ongoing star formation yielding a fairly tight correlation between our derived host ages and specific SFRs. Thus, we should keep in mind that our luminosity-weighted properties might be different from those produced with mass-weighted measurements. The details of the galaxy modeling and SED fitting method employed here can be found in Section 3 of Sullivan et al. (2006).

To select the sample with good SED fits, we first require that a unique solution be found. This reduced our initial host sample from 258 down to 209, mostly due to the rejected SED having too few photometric points. We next require SED coverage in both the UV and optical bands, further reducing our sample down to 174 hosts. At this point we examined the distribution of $\chi_{v}^{2}$ and eliminated an additional six hosts that had extreme values $\left(\chi_{v}^{2}>30\right.$; see Figure 2 and Le Borgne \& Rocca-Volmerange 2002) leaving a total sample of 168 . The 90 hosts that did not pass these criteria had no or limited optical photometry mostly due to not being members of any of the major galaxy catalogs (i.e., anonymous) and not being in the SDSS footprint which would allow us to derive the integrated flux from SDSS imaging. A subset of these also had no UV coverage because of proximity to a UV-bright star, which precludes GALEX observations due to detector safety. Table 2 lists the host T-type (de Vaucouleurs 1959) and the SED fit derived properties for the 166 unique hosts in our sample.

The number of unique hosts is two less than the number of $\mathrm{SNe}$ Ia because two galaxies hosted two $\mathrm{SNe}$ Ia each: NGC 1316 hosted SN1980N and SN1981D, and NGC 5468 hosted SN1999cp and SN2002cr. In NGC 1316, the SNe Ia produced are low stretch $(s \sim 8.5)$ with values that differ by only $3 \%$, while the two SNe Ia produced in NGC 5468 are normal stretch $(s \sim 0.98)$ and differ by $11 \%$. This is certainly not a conclusive test of using integrated host magnitudes for comparison with stretch values, but it gives no cause to doubt this method, as would be the case if these pairs of SNe Ia differed by greater amounts.

Figure 3 shows the distributions of derived host properties for the local (solid line histograms) and H09 (dot-dashed line histograms) samples. There are some interesting differences 
Table 2

Host Properties

\begin{tabular}{|c|c|c|c|c|c|c|c|c|c|c|c|}
\hline HOST & $\mathrm{T}^{\mathrm{a}}$ & Age- & $\begin{array}{l}\langle\text { Age }\rangle_{L} \\
(\log \mathrm{yr}) \\
\end{array}$ & Age+ & $M^{*}-$ & $\begin{array}{c}\left\langle M^{*}\right\rangle \\
\left(\log M_{\odot}\right) \\
\end{array}$ & $M^{*}+$ & sSFR- & $\begin{array}{c}\langle\mathrm{sSFR}\rangle \\
\left(\log \mathrm{yr}^{-1}\right)\end{array}$ & sSFR+ & $\begin{array}{c}E(B-V)_{H} \\
(\mathrm{mag}) \\
\end{array}$ \\
\hline A005629-0113 & $\ldots$ & 9.81 & 10.07 & 10.09 & 10.48 & 10.62 & 10.66 & -12.00 & -12.00 & -12.48 & 0.00 \\
\hline A011643+0047 & $\ldots$ & 8.45 & 8.71 & 8.93 & 10.06 & 10.15 & 10.20 & -9.50 & -9.31 & -9.06 & 0.20 \\
\hline A081144+4133 & $\ldots$ & 8.83 & 9.13 & 9.39 & 10.26 & 10.31 & 10.43 & -10.32 & -9.84 & -9.41 & 0.10 \\
\hline A103929+0511 & $\ldots$ & 9.51 & 9.51 & 10.05 & 10.23 & 10.26 & 10.55 & -12.00 & -10.76 & -10.71 & 0.05 \\
\hline A110932+2837 & $\ldots$ & 8.86 & 8.97 & 9.56 & 10.51 & 10.52 & 10.75 & -10.77 & -10.09 & -9.75 & 0.30 \\
\hline A112539+2249 & $\ldots$ & 8.15 & 8.62 & 9.05 & 9.15 & 9.28 & 9.36 & -9.58 & -9.23 & -8.79 & 0.05 \\
\hline A125924+2820 & $\ldots$ & 8.15 & 9.03 & 9.44 & 10.30 & 10.42 & 10.62 & -10.69 & -10.13 & -8.35 & 0.20 \\
\hline A143356+0347 & $\ldots$ & 9.51 & 9.62 & 10.09 & 9.95 & 10.03 & 10.27 & -12.00 & -11.03 & -10.69 & 0.05 \\
\hline A191131+7753 & $\ldots$ & 8.47 & 8.66 & 8.95 & 10.86 & 10.90 & 10.98 & -9.52 & -9.27 & -9.07 & 0.25 \\
\hline A215558-0104 & $\ldots$ & 9.51 & 9.55 & 9.65 & 10.25 & 10.30 & 10.38 & -12.00 & -11.62 & -10.73 & 0.00 \\
\hline CGCG180-22 & $\ldots$ & 9.04 & 9.09 & 9.28 & 10.10 & 10.13 & 10.21 & -10.41 & -10.14 & -10.06 & 0.15 \\
\hline CGCG266-031 & 3.0 & 9.15 & 9.37 & 9.61 & 10.55 & 10.62 & 10.74 & -10.83 & -10.49 & -10.17 & 0.05 \\
\hline E291-G11 & 1.0 & 8.90 & 9.73 & 10.03 & 11.16 & 11.81 & 12.27 & -11.56 & -10.85 & -9.27 & 0.30 \\
\hline E300-G09 & 5.0 & 8.50 & 8.91 & 9.59 & 9.19 & 9.72 & 10.42 & -10.56 & -9.62 & -8.50 & 0.10 \\
\hline E383-G32 & 4.5 & 8.08 & 8.35 & 8.71 & 9.70 & 10.04 & 10.27 & -9.35 & -9.00 & -8.49 & 0.25 \\
\hline E445-G66 & 1.9 & 7.00 & 7.76 & 9.15 & 10.13 & 10.51 & 11.07 & -10.37 & -8.63 & -8.14 & 0.55 \\
\hline E508-G67 & 5.0 & 8.81 & 8.90 & 9.67 & 9.92 & 10.02 & 11.06 & -11.11 & -9.88 & -8.95 & 0.00 \\
\hline E508-G75 & 3.9 & 7.83 & 9.36 & 10.03 & 10.13 & 10.76 & 11.35 & -11.57 & -10.31 & -8.14 & 0.15 \\
\hline E576-G40 & 7.0 & 7.00 & 7.30 & 8.00 & 8.59 & 8.71 & 8.90 & -8.97 & -8.66 & -8.33 & 0.45 \\
\hline IC 1277 & 6.0 & 7.00 & 7.26 & 8.21 & 10.29 & 10.44 & 10.88 & -9.26 & -8.66 & -8.15 & 0.55 \\
\hline IC3690 & 4.0 & 8.83 & 9.16 & 9.67 & 10.24 & 10.34 & 10.48 & -10.86 & -9.98 & -9.35 & 0.15 \\
\hline IC3918 & 4.4 & 8.01 & 8.28 & 8.59 & 9.27 & 9.38 & 9.45 & -9.19 & -8.94 & -8.62 & 0.15 \\
\hline IC4232 & 3.8 & 7.71 & 8.73 & 9.48 & 10.34 & 11.04 & 11.51 & -11.03 & -9.32 & -8.15 & 0.40 \\
\hline IC4423 & 4.1 & 8.88 & 9.18 & 9.72 & 10.49 & 10.62 & 10.77 & -10.89 & -10.02 & -9.49 & 0.15 \\
\hline IC4919 & 7.9 & 7.28 & 7.30 & 7.51 & 9.04 & 9.07 & 9.10 & -8.72 & -8.66 & -8.58 & 0.25 \\
\hline IC5179 & 4.0 & 8.68 & 8.86 & 8.97 & 10.21 & 10.72 & 10.83 & -10.50 & -9.41 & -8.86 & 0.35 \\
\hline $\mathrm{M}+00-06-03$ & -2.0 & 8.88 & 9.13 & 9.68 & 10.45 & 10.54 & 10.77 & -10.85 & -10.15 & -9.42 & 0.15 \\
\hline$M+01-57-14$ & 6.0 & 8.15 & 9.94 & 10.05 & 10.57 & 11.32 & 11.79 & -11.79 & -11.10 & -8.16 & 0.20 \\
\hline$M+03-27-38$ & 5.0 & 7.57 & 9.10 & 9.29 & 10.20 & 10.35 & 10.56 & -10.49 & -9.90 & -8.55 & 0.05 \\
\hline$M+05-23-37$ & 3.0 & 8.54 & 8.82 & 9.10 & 10.53 & 10.67 & 10.76 & -9.99 & -9.38 & -9.07 & 0.25 \\
\hline$M+05-54-41$ & 5.0 & 7.45 & 8.24 & 8.97 & 10.23 & 10.69 & 11.23 & -9.78 & -8.92 & -8.25 & 0.45 \\
\hline$M+06-02-17$ & 4.2 & 9.51 & 9.51 & 9.86 & 10.80 & 10.82 & 10.99 & -12.00 & -10.75 & -10.71 & 0.05 \\
\hline$M+06-06-12$ & 4.8 & 7.00 & 9.09 & 9.70 & 8.83 & 10.27 & 10.93 & -10.95 & -9.68 & -8.00 & 0.15 \\
\hline$M+06-33-20$ & -3.1 & 8.76 & 9.56 & 10.09 & 11.49 & 11.59 & 11.83 & -12.00 & -10.82 & -9.46 & 0.10 \\
\hline $\mathrm{M}+07-07-01$ & 4.2 & 7.28 & 7.81 & 8.53 & 9.66 & 9.86 & 10.04 & -9.16 & -8.63 & -8.35 & 0.30 \\
\hline$M+07-36-33$ & $\ldots$ & 8.32 & 8.92 & 9.59 & 10.03 & 10.81 & 11.42 & -11.03 & -9.57 & -8.44 & 0.20 \\
\hline$M+08-25-47$ & $\ldots$ & 8.83 & 9.16 & 9.39 & 9.96 & 9.99 & 10.16 & -10.37 & -9.98 & -9.38 & 0.05 \\
\hline$M+11-08-12$ & -2.4 & 9.51 & 10.10 & 10.11 & 10.59 & 10.82 & 10.88 & -12.00 & -12.00 & -10.71 & 0.00 \\
\hline$M+11-13-36$ & 3.3 & 8.58 & 9.15 & 9.24 & 9.67 & 9.72 & 10.18 & -10.69 & -10.18 & -8.81 & 0.00 \\
\hline M-01-04-44 & 6.0 & 7.54 & 7.98 & 8.15 & 8.91 & 8.93 & 9.20 & -8.91 & -8.63 & -8.41 & 0.25 \\
\hline M-01-09-06 & 6.0 & 8.84 & 9.08 & 9.31 & 10.51 & 10.58 & 10.77 & -10.53 & -10.14 & -9.36 & 0.05 \\
\hline M-02-02-86 & -2.0 & 9.51 & 9.51 & 10.09 & 11.67 & 11.70 & 11.97 & -12.00 & -10.76 & -10.69 & 0.10 \\
\hline M-02-16-02 & 3.0 & 7.00 & 9.03 & 10.05 & 9.29 & 9.58 & 10.50 & -11.39 & -10.13 & -8.07 & 0.05 \\
\hline M-03-01-08 & 2.5 & 8.79 & 9.05 & 9.70 & 10.17 & 10.34 & 10.95 & -10.63 & -9.81 & -9.20 & 0.05 \\
\hline M-04-12-34 & -2.6 & 8.43 & 8.79 & 9.61 & 10.64 & 10.95 & 11.14 & -11.34 & -9.81 & -8.16 & 0.55 \\
\hline M-05-28-01 & 3.7 & 7.67 & 8.28 & 9.15 & 10.48 & 10.98 & 11.41 & -10.32 & -8.94 & -8.17 & 0.45 \\
\hline NGC 105 & 1.5 & 7.04 & 9.11 & 9.59 & 9.47 & 10.87 & 11.42 & -11.04 & -9.81 & -8.03 & 0.15 \\
\hline NGC 1275 & -1.6 & 8.51 & 9.05 & 9.36 & 11.15 & 11.24 & 11.48 & -10.49 & -9.81 & -9.03 & 0.15 \\
\hline NGC 1309 & 4.0 & 7.82 & 8.47 & 8.84 & 9.78 & 9.94 & 10.64 & -10.27 & -9.08 & -8.00 & 0.25 \\
\hline NGC 1316 & -1.9 & 10.07 & 10.11 & 10.11 & 11.75 & 11.79 & 11.80 & -12.00 & -12.00 & -13.75 & 0.00 \\
\hline NGC 1371 & 1.0 & 9.51 & 9.70 & 9.77 & 10.79 & 10.90 & 11.02 & -10.60 & -10.46 & -10.15 & 0.15 \\
\hline NGC 1380 & -1.9 & 10.05 & 10.10 & 10.10 & 11.27 & 11.33 & 11.36 & -12.00 & -12.00 & -13.27 & 0.05 \\
\hline NGC 1448 & 5.9 & 8.95 & 9.37 & 9.40 & 10.22 & 10.69 & 10.73 & -10.19 & -9.90 & -9.40 & 0.20 \\
\hline NGC 1699 & 3.0 & 8.71 & 9.59 & 9.62 & 10.12 & 10.37 & 10.41 & -10.46 & -10.22 & -9.24 & 0.00 \\
\hline NGC 191A & -2.0 & 9.51 & 10.10 & 10.11 & 10.76 & 11.00 & 11.02 & -12.00 & -12.00 & -10.70 & 0.00 \\
\hline NGC 2076 & -0.6 & 9.18 & 9.67 & 9.77 & 9.62 & 10.02 & 10.14 & -11.12 & -10.41 & -9.62 & 0.15 \\
\hline NGC 2258 & -2.0 & 8.43 & 8.91 & 9.48 & 11.43 & 11.52 & 12.33 & -11.01 & -10.03 & -8.40 & 0.50 \\
\hline NGC 2441 & 3.1 & 8.71 & 9.12 & 9.40 & 10.31 & 10.66 & 10.87 & -10.04 & -9.70 & -9.28 & 0.25 \\
\hline NGC 252 & -1.0 & 8.94 & 9.43 & 9.90 & 10.87 & 11.25 & 11.90 & -11.44 & -10.56 & -9.43 & 0.25 \\
\hline
\end{tabular}


Table 2

(Continued)

\begin{tabular}{|c|c|c|c|c|c|c|c|c|c|c|c|}
\hline HOST & $\mathrm{T}^{\mathrm{a}}$ & Age- & $\begin{array}{r}\langle\text { Age }\rangle_{L} \\
(\log \mathrm{yr}) \\
\end{array}$ & Age+ & $M^{*}-$ & $\begin{array}{c}\left\langle M^{*}\right\rangle \\
\left(\log M_{\odot}\right) \\
\end{array}$ & $M^{*}+$ & sSFR- & $\begin{array}{c}\langle\mathrm{sSFR}\rangle \\
\left(\log \mathrm{yr}^{-1}\right)\end{array}$ & sSFR+ & $\begin{array}{c}E(B-V)_{H} \\
(\mathrm{mag}) \\
\end{array}$ \\
\hline NGC 2935 & 3.2 & 7.58 & 8.25 & 10.05 & 10.68 & 10.94 & 11.31 & -12.00 & -8.61 & -8.23 & 0.55 \\
\hline NGC 2962 & -1.0 & 8.63 & 8.93 & 10.11 & 10.10 & 10.20 & 10.50 & -12.00 & -10.05 & -8.95 & 0.35 \\
\hline NGC 3021 & 4.3 & 8.71 & 8.90 & 9.19 & 9.76 & 9.87 & 9.97 & -9.88 & -9.45 & -9.23 & 0.30 \\
\hline NGC 3147 & 3.9 & 8.98 & 9.34 & 9.63 & 10.75 & 11.31 & 11.66 & -11.02 & -10.15 & -9.41 & 0.15 \\
\hline NGC 3169 & 1.2 & 9.04 & 9.19 & 9.43 & 10.64 & 10.70 & 10.79 & -10.59 & -10.23 & -10.07 & 0.15 \\
\hline NGC 3190 & 1.0 & 9.51 & 10.11 & 10.11 & 10.55 & 10.83 & 10.89 & -12.00 & -12.00 & -10.62 & 0.00 \\
\hline NGC 3327 & 3.0 & 8.79 & 9.18 & 9.75 & 10.66 & 10.77 & 10.96 & -10.92 & -10.02 & -9.36 & 0.15 \\
\hline NGC 3332 & -3.0 & 8.72 & 8.96 & 10.11 & 11.11 & 11.15 & 11.51 & -12.00 & -10.07 & -9.29 & 0.30 \\
\hline NGC 3368 & 2.0 & 8.80 & 9.22 & 9.83 & 11.09 & 11.26 & 11.54 & -11.01 & -10.30 & -9.59 & 0.25 \\
\hline NGC 3370 & 5.3 & 8.00 & 8.55 & 9.08 & 9.55 & 9.69 & 9.83 & -9.97 & -9.15 & -8.63 & 0.25 \\
\hline NGC 3663 & 3.5 & 8.53 & 9.08 & 9.44 & 10.04 & 10.81 & 11.21 & -11.00 & -9.60 & -8.54 & 0.30 \\
\hline NGC 3672 & 5.0 & 7.92 & 8.65 & 8.97 & 10.04 & 10.23 & 10.34 & -9.52 & -9.23 & -8.58 & 0.35 \\
\hline NGC 382 & -5.0 & 9.13 & 10.05 & 10.05 & 10.17 & 11.54 & 11.56 & -11.80 & -11.24 & -9.63 & 0.45 \\
\hline NGC 3873 & -5.0 & 9.51 & 10.00 & 10.11 & 10.93 & 11.13 & 11.23 & -12.00 & -12.00 & -10.68 & 0.00 \\
\hline NGC 3987 & 3.0 & 9.08 & 9.30 & 9.77 & 10.69 & 10.78 & 10.97 & -10.93 & -10.43 & -10.08 & 0.25 \\
\hline NGC 4038 & 8.8 & 7.43 & 7.79 & 8.15 & 9.85 & 10.05 & 10.17 & -8.95 & -8.64 & -8.38 & 0.30 \\
\hline NGC 4055 & -5.0 & 9.51 & 10.11 & 10.11 & 11.11 & 11.39 & 11.42 & -12.00 & -12.00 & -10.66 & 0.00 \\
\hline NGC 4172 & 2.7 & 9.51 & 9.51 & 10.11 & 11.26 & 11.28 & 11.54 & -12.00 & -10.76 & -10.72 & 0.10 \\
\hline NGC 4321 & 4.4 & 8.57 & 9.17 & 9.26 & 10.74 & 10.81 & 11.09 & -10.57 & -10.22 & -9.01 & 0.00 \\
\hline NGC 4493 & -4.0 & 8.90 & 9.61 & 10.11 & 10.93 & 11.04 & 11.23 & -12.00 & -10.88 & -9.87 & 0.10 \\
\hline NGC 4495 & 1.9 & 8.92 & 9.16 & 9.19 & 10.39 & 10.50 & 10.53 & -9.72 & -9.68 & -9.54 & 0.30 \\
\hline NGC 4501 & 3.0 & 8.36 & 8.79 & 9.24 & 10.91 & 11.03 & 11.23 & -10.53 & -9.45 & -8.86 & 0.35 \\
\hline NGC 4527 & 3.8 & 8.62 & 9.30 & 9.56 & 10.60 & 10.78 & 10.91 & -10.79 & -10.43 & -9.03 & 0.20 \\
\hline NGC 4536 & 4.5 & 9.00 & 9.18 & 9.36 & 10.42 & 10.47 & 10.68 & -10.45 & -10.02 & -9.38 & 0.05 \\
\hline NGC 4619 & 3.1 & 8.44 & 8.88 & 9.08 & 10.87 & 10.92 & 11.11 & -9.97 & -9.56 & -9.01 & 0.15 \\
\hline NGC 4639 & 3.8 & 9.16 & 9.32 & 9.46 & 10.07 & 10.14 & 10.24 & -10.42 & -10.12 & -9.63 & 0.05 \\
\hline NGC 4675 & 3.0 & 8.56 & 9.62 & 9.77 & 10.14 & 10.42 & 10.43 & -10.93 & -10.26 & -9.10 & 0.10 \\
\hline NGC 4680 & PEC & 8.18 & 8.76 & 9.05 & 10.11 & 10.24 & 10.33 & -9.92 & -9.32 & -8.81 & 0.30 \\
\hline NGC 4704 & 3.5 & 8.35 & 8.95 & 9.25 & 10.55 & 10.59 & 10.82 & -10.31 & -9.67 & -8.95 & 0.15 \\
\hline NGC 5018 & -5.0 & 9.51 & 9.51 & 9.61 & 11.21 & 11.34 & 11.37 & -11.24 & -10.76 & -10.60 & 0.10 \\
\hline NGC 5061 & -5.0 & 9.51 & 9.61 & 9.61 & 10.78 & 10.85 & 10.87 & -10.92 & -10.88 & -10.67 & 0.05 \\
\hline NGC 5185 & 3.0 & 9.18 & 9.37 & 9.61 & 10.89 & 10.94 & 11.06 & -10.83 & -10.49 & -10.20 & 0.10 \\
\hline NGC 523 & 4.2 & 8.95 & 9.32 & 9.51 & 10.23 & 10.38 & 10.53 & -10.71 & -10.45 & -9.56 & 0.00 \\
\hline NGC 524 & -1.0 & 10.05 & 10.10 & 10.10 & 11.82 & 11.89 & 11.91 & -12.00 & -12.00 & -13.82 & 0.05 \\
\hline NGC 5468 & 6.0 & 7.18 & 7.43 & 7.87 & 9.39 & 9.48 & 9.77 & -9.04 & -8.65 & -8.25 & 0.20 \\
\hline NGC 5584 & 6.0 & 8.27 & 8.71 & 8.92 & 9.69 & 9.82 & 9.87 & -9.49 & -9.31 & -8.94 & 0.15 \\
\hline NGC 6038 & 5.0 & 8.91 & 9.18 & 9.34 & 10.94 & 10.99 & 11.03 & -10.29 & -10.02 & -9.60 & 0.10 \\
\hline NGC 6063 & 6.0 & 8.56 & 8.95 & 9.21 & 9.85 & 9.89 & 10.11 & -10.11 & -9.67 & -9.10 & 0.10 \\
\hline NGC 6104 & $\ldots$ & 8.92 & 9.16 & 9.19 & 10.71 & 10.83 & 10.86 & -9.90 & -9.68 & -9.51 & 0.20 \\
\hline NGC 632 & -1.5 & 7.89 & 9.24 & 9.35 & 9.83 & 10.00 & 10.13 & -10.57 & -10.34 & -8.43 & 0.00 \\
\hline NGC 6365A & 5.9 & 8.43 & 9.40 & 9.59 & 10.44 & 10.74 & 10.87 & -10.26 & -9.93 & -9.02 & 0.05 \\
\hline NGC 6411 & -5.0 & 9.51 & 9.70 & 10.11 & 10.79 & 10.91 & 11.13 & -12.00 & -12.00 & -10.67 & 0.05 \\
\hline NGC 6627 & 3.0 & 8.88 & 9.42 & 9.78 & 10.68 & 10.85 & 11.08 & -12.00 & -10.56 & -9.44 & 0.05 \\
\hline NGC 6685 & -3.0 & 8.72 & 8.96 & 10.11 & 10.73 & 10.76 & 11.12 & -12.00 & -10.07 & -9.31 & 0.30 \\
\hline NGC 673 & 5.0 & 7.81 & 8.13 & 8.48 & 10.24 & 10.37 & 10.78 & -9.39 & -8.84 & -8.32 & 0.30 \\
\hline NGC 6916 & 4.0 & 8.69 & 9.59 & 9.62 & 10.43 & 10.68 & 10.72 & -10.46 & -10.22 & -9.22 & 0.00 \\
\hline NGC 6928 & 2.0 & 8.15 & 8.83 & 8.97 & 11.05 & 11.17 & 11.40 & -10.37 & -9.93 & -8.26 & 0.40 \\
\hline NGC 6949 & 5.0 & 7.00 & 8.17 & 9.37 & 9.09 & 9.64 & 10.42 & -10.02 & -8.86 & -8.04 & 0.10 \\
\hline NGC 6951 & 4.0 & 7.88 & 8.63 & 9.61 & 10.84 & 10.96 & 11.32 & -10.94 & -9.05 & -8.18 & 0.35 \\
\hline NGC 6962 & 2.0 & 8.83 & 9.30 & 9.56 & 10.96 & 11.09 & 11.23 & -10.79 & -10.43 & -9.67 & 0.10 \\
\hline NGC 7311 & 2.0 & 8.94 & 9.32 & 9.56 & 10.84 & 10.97 & 11.09 & -10.78 & -10.45 & -9.94 & 0.10 \\
\hline NGC 7541 & 4.0 & 7.18 & 8.48 & 9.70 & 10.29 & 10.66 & 10.98 & -10.82 & -9.11 & -8.22 & 0.35 \\
\hline NGC 7678 & 5.0 & 8.67 & 8.74 & 9.05 & 10.04 & 10.40 & 10.75 & -10.37 & -9.38 & -8.81 & 0.15 \\
\hline NGC 7780 & 2.0 & 8.94 & 9.30 & 9.61 & 10.22 & 10.35 & 10.48 & -10.87 & -10.43 & -9.93 & 0.15 \\
\hline NGC 809 & -1.0 & 9.51 & 9.51 & 9.61 & 10.66 & 10.70 & 10.78 & -10.99 & -10.75 & -10.69 & 0.05 \\
\hline NGC 976 & 5.0 & 8.66 & 9.01 & 9.46 & 10.72 & 10.78 & 10.99 & -10.60 & -9.77 & -9.16 & 0.15 \\
\hline PGC0059076 & $\ldots$ & 8.64 & 8.98 & 9.13 & 9.80 & 9.84 & 10.00 & -10.08 & -9.72 & -9.14 & 0.05 \\
\hline UGC 10030 & 3.0 & 8.88 & 9.27 & 9.77 & 10.89 & 10.97 & 11.19 & -10.94 & -10.16 & -9.47 & 0.10 \\
\hline UGC 10244 & 3.7 & 8.62 & 9.07 & 9.76 & 10.41 & 10.46 & 10.65 & -10.88 & -9.86 & -9.15 & 0.25 \\
\hline
\end{tabular}


Table 2

(Continued)

\begin{tabular}{|c|c|c|c|c|c|c|c|c|c|c|c|}
\hline HOST & $\mathrm{T}^{\mathrm{a}}$ & Age- & $\begin{array}{r}\langle\text { Age }\rangle_{L} \\
(\log \mathrm{yr}) \\
\end{array}$ & Age+ & $M^{*-}$ & $\begin{array}{c}\left\langle M^{*}\right\rangle \\
\left(\log M_{\odot}\right) \\
\end{array}$ & $M^{*}+$ & sSFR- & $\begin{array}{c}\langle\text { sSFR }\rangle \\
\left(\log \mathrm{yr}^{-1}\right)\end{array}$ & sSFR+ & $\begin{array}{c}E(B-V)_{H} \\
(\mathrm{mag})\end{array}$ \\
\hline UGC 10743 & 1.0 & 7.88 & 8.51 & 9.77 & 9.93 & 10.56 & 11.18 & -11.42 & -9.13 & -8.08 & 0.70 \\
\hline UGC 1087 & 5.0 & 8.69 & 9.26 & 9.48 & 10.04 & 10.20 & 10.29 & -10.14 & -9.77 & -9.25 & 0.10 \\
\hline UGC 11149 & $\ldots$ & 7.98 & 9.61 & 10.09 & 11.17 & 11.72 & 12.59 & -12.00 & -12.00 & -8.00 & 0.05 \\
\hline UGC 11723 & 3.0 & 9.01 & 9.50 & 9.87 & 10.22 & 10.41 & 10.58 & -11.05 & -10.65 & -9.62 & 0.15 \\
\hline UGC 1333 & 3.0 & 8.99 & 9.05 & 9.24 & 11.20 & 11.25 & 11.34 & -10.34 & -10.13 & -10.01 & 0.20 \\
\hline UGC 14 & 5.5 & 7.86 & 8.56 & 9.25 & 10.52 & 10.70 & 10.87 & -10.20 & -9.16 & -8.59 & 0.35 \\
\hline UGC 2384 & 3.9 & 7.40 & 8.54 & 9.74 & 10.66 & 11.59 & 12.43 & -11.42 & -9.15 & -8.00 & 0.70 \\
\hline UGC 2650 & 2.0 & 8.50 & 9.98 & 10.11 & 10.66 & 11.96 & 12.33 & -12.00 & -11.11 & -8.07 & 0.45 \\
\hline UGC 2708 & -2.0 & 8.95 & 9.86 & 10.00 & 10.81 & 11.08 & 11.19 & -12.00 & -12.00 & -9.98 & 0.00 \\
\hline UGC 272 & 6.5 & 8.00 & 8.46 & 8.99 & 9.41 & 9.54 & 9.68 & -9.69 & -9.07 & -8.68 & 0.20 \\
\hline UGC 2829 & -2.0 & 8.99 & 9.86 & 10.01 & 10.73 & 10.97 & 11.06 & -12.00 & -12.00 & -10.09 & 0.00 \\
\hline UGC 3151 & 5.0 & 8.70 & 9.21 & 9.77 & 10.53 & 10.65 & 10.92 & -12.00 & -10.06 & -9.16 & 0.10 \\
\hline UGC 3218 & 3.0 & 7.00 & 7.34 & 7.75 & 10.09 & 10.19 & 10.35 & -8.93 & -8.65 & -8.39 & 0.35 \\
\hline UGC 3725 & -3.0 & 9.49 & 9.99 & 10.10 & 11.28 & 12.24 & 12.34 & -12.00 & -11.18 & -9.80 & 0.50 \\
\hline UGC 3770 & 10.0 & 7.00 & 7.51 & 7.93 & 9.65 & 9.82 & 10.10 & -9.11 & -8.65 & -8.18 & 0.40 \\
\hline UGC 3845 & 3.9 & 7.00 & 7.43 & 7.65 & 9.16 & 9.30 & 9.39 & -8.89 & -8.65 & -8.41 & 0.20 \\
\hline UGC 4008 & 0.0 & 7.57 & 9.30 & 9.58 & 10.85 & 10.98 & 11.12 & -10.80 & -10.43 & -8.54 & 0.10 \\
\hline UGC 4195 & 3.0 & 8.65 & 9.21 & 9.25 & 10.35 & 10.50 & 10.54 & -9.97 & -9.72 & -9.23 & 0.25 \\
\hline UGC 4322 & -5.0 & 8.07 & 8.31 & 9.89 & 10.64 & 11.12 & 12.02 & -12.00 & -8.61 & -8.04 & 0.70 \\
\hline UGC 4414 & 0.0 & 9.09 & 9.46 & 9.56 & 10.86 & 10.95 & 11.06 & -10.78 & -10.64 & -10.11 & 0.10 \\
\hline UGC 4455 & 1.0 & 8.66 & 9.22 & 9.67 & 10.61 & 10.76 & 10.90 & -10.78 & -9.96 & -9.17 & 0.15 \\
\hline UGC 4614 & $\ldots$ & 7.23 & 8.32 & 9.21 & 9.96 & 10.32 & 10.49 & -9.94 & -8.97 & -8.31 & 0.30 \\
\hline UGC 5129 & 1.0 & 8.62 & 8.98 & 9.35 & 10.11 & 10.22 & 10.34 & -10.59 & -9.72 & -9.09 & 0.15 \\
\hline UGC 52 & 5.0 & 8.19 & 9.32 & 9.49 & 10.24 & 10.38 & 10.56 & -10.74 & -10.45 & -8.76 & 0.00 \\
\hline UGC 5234 & 5.0 & 7.51 & 8.90 & 9.42 & 10.15 & 10.55 & 10.80 & -10.62 & -9.44 & -8.34 & 0.25 \\
\hline UGC 5378 & 3.0 & 7.20 & 8.28 & 9.21 & 9.52 & 9.88 & 10.09 & -10.19 & -8.94 & -8.27 & 0.35 \\
\hline UGC 5542 & -5.0 & 9.51 & 9.61 & 10.05 & 10.92 & 10.99 & 11.20 & -12.00 & -10.88 & -10.68 & 0.05 \\
\hline UGC 7934 & 2.8 & 8.67 & 9.10 & 9.36 & 10.29 & 10.46 & 10.57 & -10.33 & -9.63 & -9.19 & 0.20 \\
\hline UGC 8162 & 6.0 & 8.07 & 8.56 & 9.21 & 9.93 & 10.06 & 10.21 & -9.75 & -9.18 & -8.78 & 0.15 \\
\hline UGC 9391 & 8.0 & 8.33 & 8.76 & 9.22 & 8.47 & 8.60 & 8.74 & -9.78 & -9.36 & -8.96 & 0.05 \\
\hline UGC 9612 & 5.0 & 7.45 & 8.44 & 9.20 & 10.43 & 10.70 & 10.89 & -10.12 & -9.06 & -8.43 & 0.30 \\
\hline UGC 9640 & -5.0 & 9.51 & 9.70 & 10.05 & 11.39 & 11.48 & 11.65 & -12.00 & -12.00 & -10.72 & 0.05 \\
\hline
\end{tabular}

Note. ${ }^{\text {a }}$ Numerical type according to de Vaucouleurs (1959).

between the two samples. The discrepancy in the mass distributions (Figure 3(b)) can be explained because local $\mathrm{SNe}$ are discovered in host-targeted surveys which prefer more massive hosts, while the SNLS is an areal survey without a mass bias and therefore includes lower-mass hosts. The discrepancy in the SFR distributions (Figure 3(c)) could be due to a luminosity bias from the host-targeted low-redshift surveys. Here the higher-redshift areal survey does not prefer luminous, and therefore higher SFR, hosts. These differences are reinforced if there is a correlation between mass and SFR. In this case, the same bias that produces the deficit of low-mass hosts in the local mass distribution produces the deficit of lower SFR hosts in the local SFR distribution. Figure 3(d) shows that when considering the specific star formation (sSFR), defined as SFR divided by stellar mass, our distributions are fairly similar. Thus, from here on we consider sSFR in preference over SFR.

\section{RESULTS}

\subsection{Host Properties versus SN Light-curve Stretch}

We start by examining correlations in host properties with SN Ia light-curve stretch. In the following discussion, it is good to keep in mind that higher-stretch SNe Ia are brighter and lower-stretch SNe Ia are fainter. In these plots, we highlight the transition between the low-luminosity (i.e., low-stretch) and normal SNe with an horizontal dashed line at $s=0.80$.

Figure 4 plots the log of the sSFR against hosted SN Ia lightcurve stretch. For this plot and plots following, the symbols indicate the host's sSFR: filled circles indicate hosts with no detected star formation ( $\mathrm{sSFR} \leqslant 10^{-12} \mathrm{yr}^{-1}$ ), filled stars indicate strongly star-forming hosts (sSFR $>10^{-9.5} \mathrm{yr}^{-1}$ ), and filled squares indicate hosts with intermediate sSFR. Typically, one would expect elliptical galaxies to belong in the lowest sSFR group and spiral galaxies to belong to the intermediate and high sSFR groups. Our aim here is to use a metric that is more physical than morphology; hence we do not use morphological classifications to examine these SN Ia hosts.

We notice several interesting features in this diagram. We see that the sensitivity threshold of our models is just above $\sim 10^{-12} \mathrm{yr}^{-1}$. We set all hosts with no detected SSFR to this value and consider these to be "dead" hosts. At the high end, we see the timescale limit which is determined by the lifetime of stars with SEDs that peak in the UV, $\sim 3 \times 10^{8} \mathrm{yr}$. Since all of our hosts are essentially at zero redshift, this limit appears as a line. The triangles show the average stretch in three sSFR bins indicated by the vertical dashed lines. Note that low-stretch 

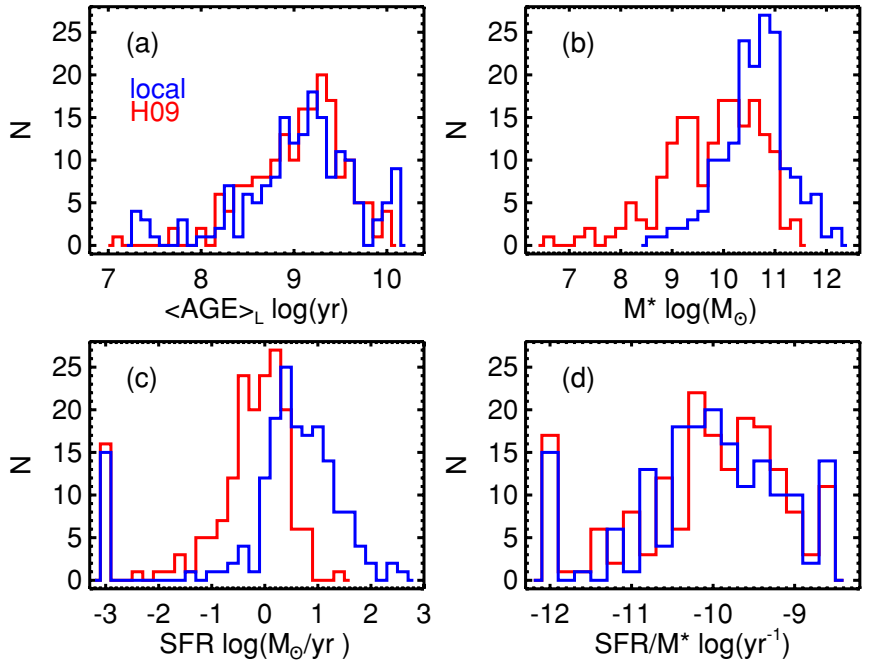

Figure 3. Frequency diagrams of host properties derived from SED fitting using the PEGASE.2 library (Le Borgne et al. 2004) with the SNLS sample from H09 as the dash-dotted line histograms and the local SN Ia light-curve fit sample as the solid line histograms: (a) luminosity-weighted age, (b) stellar mass, (c) SFR, and (d) sSFR defined as SFR/M*. Panel (c) illustrates our SFR threshold of $10^{-3} M_{\odot} \mathrm{yr}^{-1}$. Panel (d) illustrates our sSFR thresshold of $10^{-12} \mathrm{yr}^{-1}$. Hosts below these values are set to the threshold value. We see that the local sample is missing the low-mass and intermediate SFR peaks seen in the H09 sample. In sSFR, the two distribution are quite similar.

(A color version of this figure is available in the online journal.)

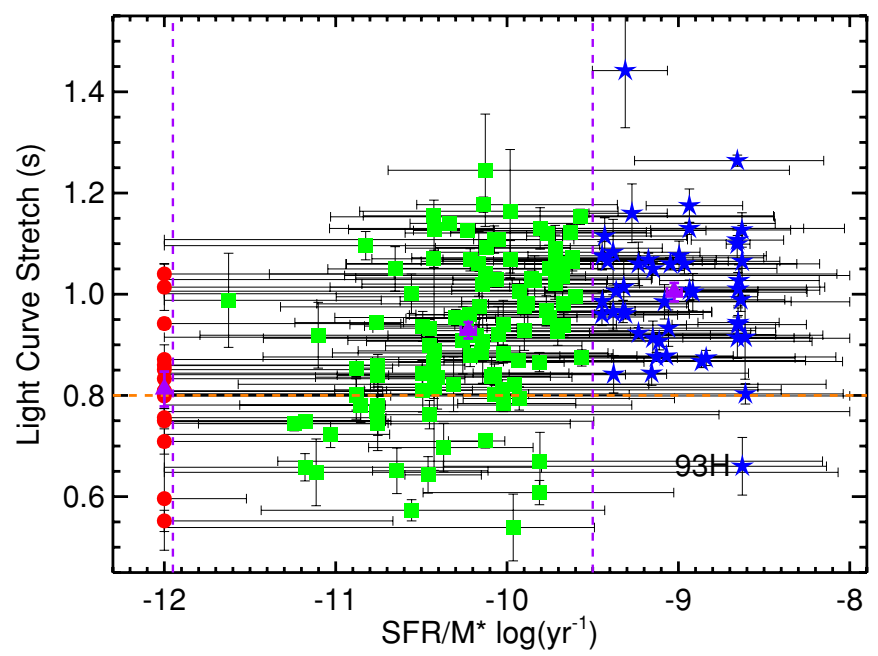

Figure 4. Specific SFR as derived from PEGASE. 2 SED fits plotted as a function of light-curve stretch for SN Ia hosts. Filled circles indicate hosts with very low specific star formation ( $\mathrm{sSFR} \leqslant 10^{-12} \mathrm{yr}^{-1}$ ), filled stars indicate hosts with strong specific star formation ( $\mathrm{sSFR}>10^{-9.5} \mathrm{yr}^{-1}$ ), and filled squares indicate hosts with intermediate sSFR. Averages for the three sSFR bins (vertical dashed lines) are shown as the filled triangles with the error bars indicating the error in the mean. The division between normal and low-stretch $\mathrm{SNe}$ is indicated by the horizontal dashed line at $s=0.8$. The one high sSFR host of a low-stretch SN Ia (SN 1993H) is indicated. Stretch, on average, appears to increase with host sSFR.

(A color version of this figure is available in the online journal.)

SNe are rare in high sSFR hosts (the one exception is $\mathrm{SN} 1993 \mathrm{H}$, as indicated in the plot), but common in intermediate and low sSFR hosts.

Figure 5 plots the luminosity-weighted host age against stretch, with the same symbol coding as in Figure 4. This plot shows that age and sSFR are well correlated with all the high sSFR galaxies having ages less than 1 Gyr and all the low sSFR hosts having ages greater than $4 \mathrm{Gyr}$ as expected (see

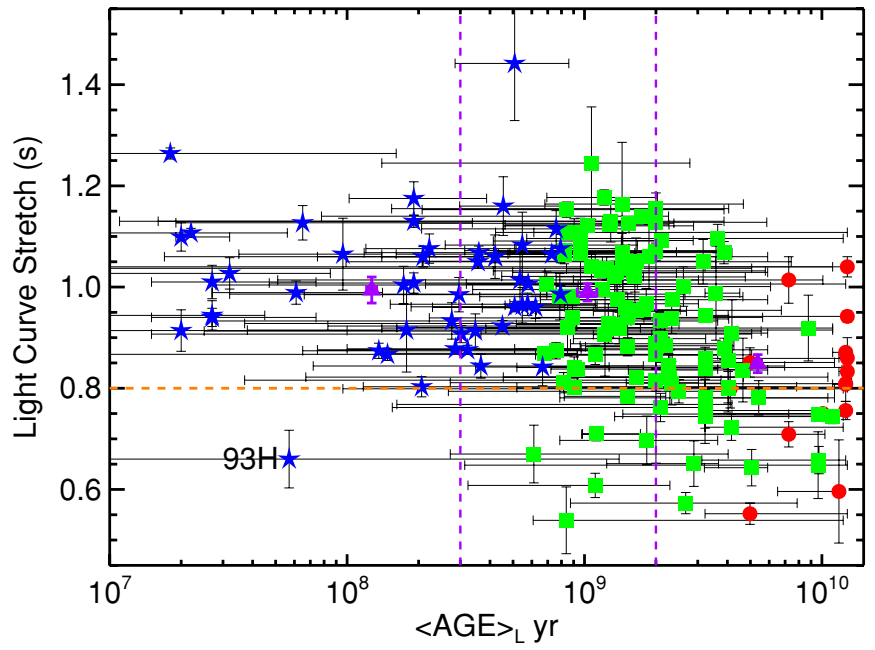

Figure 5. Luminosity-weighted stellar age as derived from PEGASE.2 SED fits plotted as a function of light-curve stretch for SN Ia hosts. The host sSFR is coded as in Figure 4. The average in three age bins divided at $3 \times 10^{8}$ and $2 \times 10^{9} \mathrm{yr}$ (vertical dashed lines) is shown by the filled triangles. The horizontal dashed line indicates the division between normal and low-stretch SNe. The one low-stretch SN Ia with a luminosity-weighted age less than $3 \times 10^{8} \mathrm{yr}$ (SN $1993 \mathrm{H}$ ) is indicated. After a host exceeds $1 \mathrm{Gyr}$ in age, it begins producing progressively lower-stretch $\mathrm{SNe} \mathrm{Ia}$, on average.

(A color version of this figure is available in the online journal.)

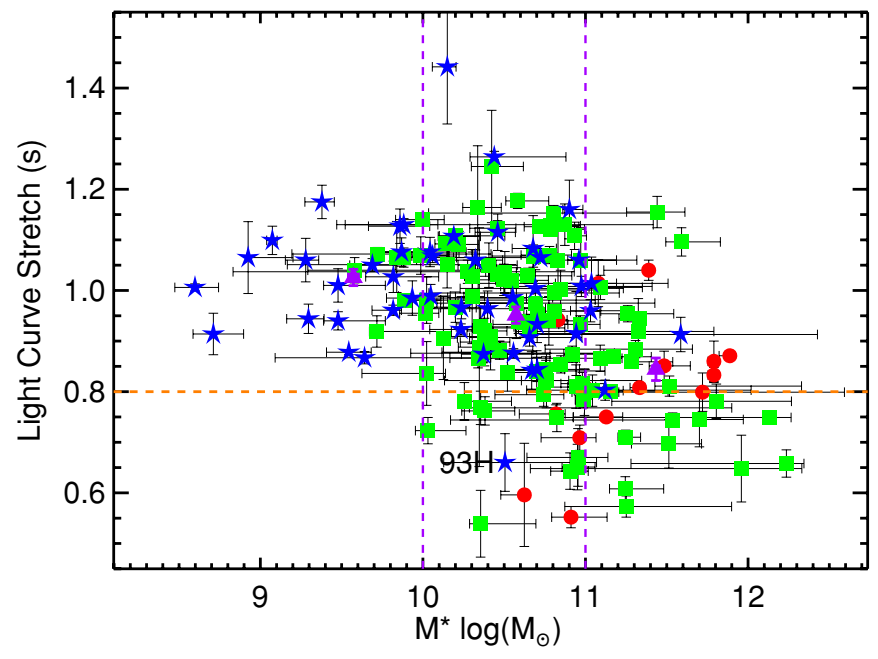

Figure 6. Stellar mass as derived from PEGASE.2 SED fits plotted as a function of light-curve stretch for SN Ia hosts. The host sSFR is coded as in Figure 4. The averages of the sample divided at $\log M_{*}=10$ and 11 (vertical dashed lines) are shown by the filled triangles. There are no hosts of low-stretch $\mathrm{SNe}$ with masses less than $10^{10} M_{\odot}$. The exceptional SN $1993 \mathrm{H}$ is indicated (see the text). This figure is very similar to Figure 4 in H09 derived from a higher-redshift sample, but here we have a larger sample of low-stretch SNe Ia.

(A color version of this figure is available in the online journal.)

Section 2.2). The averages (filled triangles) were derived from three age bins (divided at $3 \times 10^{8}$ and $2 \times 10^{9} \mathrm{yr}$ ) and show a downward trend of stretch with age beyond 1 Gyr. Here we find the low-stretch SNe to be evenly distributed between host luminosity-weighted ages older than $5 \times 10^{8} \mathrm{yr}$, but rare below this age with SN $1993 \mathrm{H}$ again being the sole exception.

Figure 6 plots host stellar mass, $M_{*}$, against light-curve stretch and shows that hosts of the lowest stretch $\mathrm{SNe}$ Ia tend to have stellar masses higher than $10^{10} M_{\odot}$. The higher-stretch $\mathrm{SNe}$ Ia hosts have a much wider mass range $\left(10^{8}-10^{12} M_{\odot}\right)$. 


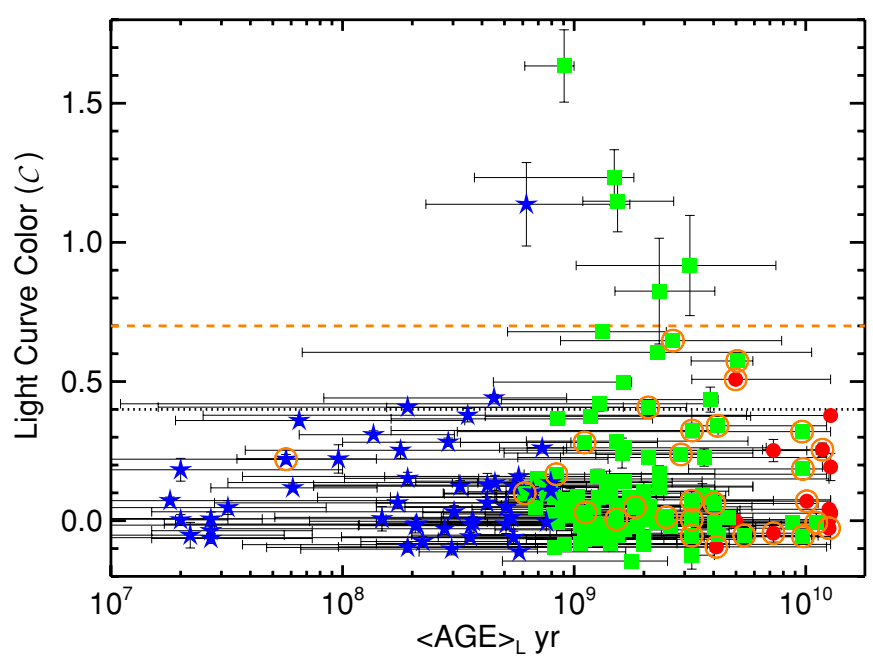

Figure 7. Luminosity-weighted age as derived from PEGASE.2 SED fits plotted as a function of SN color for the local SN Ia hosts. The host sSFR is coded as in Figure 4. The SNe with stretch less than $s=0.80$ are overplotted with open circles. The two horizontal lines illustrate two color cuts applied to our sample: the dashed line at $\mathcal{C}=0.7$ is our cut for defining red SNe Ia, and the dotted line at $\mathcal{C}=0.4$ is our cut for deriving $\mathrm{Ni}$ masses (see the text). There is no obvious correlation; however, all of the reddest $\mathrm{SNe}$ are hosted by galaxies with luminosity-weighted ages near $1 \mathrm{Gyr}$. It is also clear that the lowest stretch $\mathrm{SNe}$ are not the reddest $\mathrm{SNe}$ in our sample.

(A color version of this figure is available in the online journal.)

\subsection{SN Ia Light-curve Color}

There is a major challenge in interpreting SN Ia peak color because of the unknown mix of two potential sources of the color: intrinsic SN Ia color from the details of the explosion itself and host galaxy line-of-sight reddening. In other words, the host extinction is not accounted for in the fitting process, which means that the peak color contains an intrinsic component and a host extinction component. This difficulty manifests itself when the color-luminosity relation is converted into a dust extinction law. In most cases, the resulting $R_{V}$ is much lower than what is found in the Milky Way or other galaxies (Tripp 1998). However, it is clearly not appropriate to assume Milky-Way-like dust is responsible for SN Ia residual color (Conley et al. 2007).

There is potentially important information about the SN Ia explosion contained in the intrinsic color, if we could quantify and remove the line of sight, or host extinction part. Unfortunately, there are no observable signatures that allow these two sources to be disentangled, although extending the light-curve data to the near infrared may minimize the problem (Kasen 2006; Wood-Vasey et al. 2008). Our host data show no correlations between host proporties and SN peak color. The only feature worth noting, shown in Figure 7, is that all of the reddest $\mathrm{SNe}$ appear in hosts with intermediate age $(\sim 1 \mathrm{Gyr})$. The low-stretch SNe with $s<0.80$, indicated in the plot with the large open circles, are not the reddest $\mathrm{SNe}$ as might be expected from the color-luminosity relation (Tripp 1998; Tripp \& Branch 1999; Parodi et al. 2000).

Another way to examine this issue is to look at $\mathrm{SN}$ color as a function of host extinction, as shown in Figure 8. We see an average trend in SSFR with host extinction such that hosts with higher sSFR also have higher host extinction, a finding that gives us encouragement that the extinction estimates from PEGASE.2 are robust. The average $\mathrm{SN}$ peak color (filled triangles) shows no trend with host extinction, and the reddest $\mathrm{SNe}$ appear in hosts covering a substantial range of host extinctions. This could be

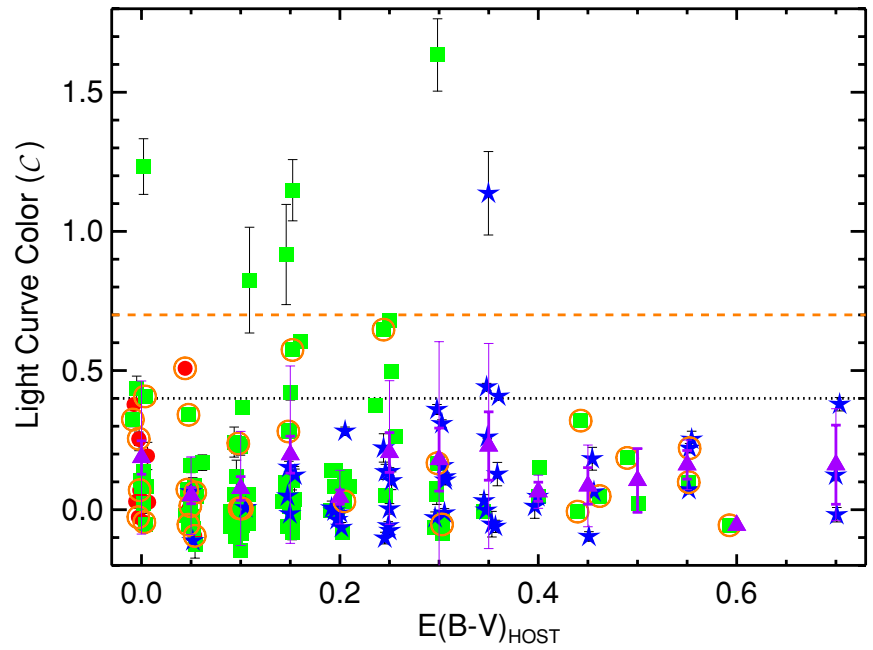

Figure 8. $\mathrm{SN}$ color as a function of average host color excess, $E(B-V)_{\text {HOST }}$, as derived from PEGASE.2 SED fits for the local SN Ia hosts. The host sSFR is coded as in Figure 4. The SNe with stretch less than $s=0.80$ are overplotted with open circles, and the color cut lines are the same as in Figure 7. To better illustrate the distributions in each discrete bin of $E(B-V)_{\text {HOST }}(0.05 \mathrm{Mag})$, a small random offset was added to each point. The average SN color in each host extinction bin is shown by the filled triangles. Note that the sSFR goes up with host extinction, as expected, and that the reddest SNe appear in hosts with a range of host extinctions.

(A color version of this figure is available in the online journal.)

due to the clumpiness of host extinction which makes an average host extinction irrelevant for the specific line of sight to the SN.

\subsection{A Subsample with Low Host Extinction}

In an effort to mitigate the difficulty of untangling host extinction and intrinsic SN color, Gallagher et al. (2008) made a study of local SNe Ia in hosts that were morphologically classified as early-type and thus thought to be low-extinction hosts. They used spectroscopic line diagnostics to determine metallicities and ages. We have the opportunity to select hosts with low extinction inferred from our SED fits, without the assumption that early-type galaxies all have low extinction and without possible host classification errors.

To test the impact of host extinction on cosmological fitting, we look for residual trends in the cosmologically corrected peak SN Ia brightness as a function of host luminosity-weighted age. We calculate the peak absolute $B$-band brightness for our $\mathrm{SNe}$ Ia, corrected for stretch and color using

$$
M_{B}=m_{B \max }+\alpha(s-1.0)-\beta \mathcal{C}-\mu_{B}\left(z_{\text {spec }}\right),
$$

where $\alpha=1.2, \beta=2.9$ (Sullivan et al. 2009), and $\mu_{B}\left(z_{\mathrm{spec}}\right)$ is the distance modulus based on the spectroscopic redshift (A. Conley et al. 2010, in preparation). Since we are testing cosmological fitting, we eliminate low-stretch $(s<0.75)$ and $\operatorname{red}(\mathcal{C}>0.7) \mathrm{SNe}$ Ia. The distance modulus, $\mu_{B}$, is only accurate if the SNe Ia are in the Hubble flow so we also eliminate hosts with $z<0.013$. After applying these cuts, we are left with a sample of $85 \mathrm{SNe}$ Ia.

We plot the stretch-color-corrected absolute peak $B$ magnitude against the luminosity-weighted host age for this sample in the bottom panel of Figure 9 using the previously defined symbols based on sSFR. In the top panel of Figure 9, we plot the same values for a low host extinction subsample $(N=22)$, with $E(B-V)_{\text {HOST }} \leqslant 0.05$ according to our SED fits. The 


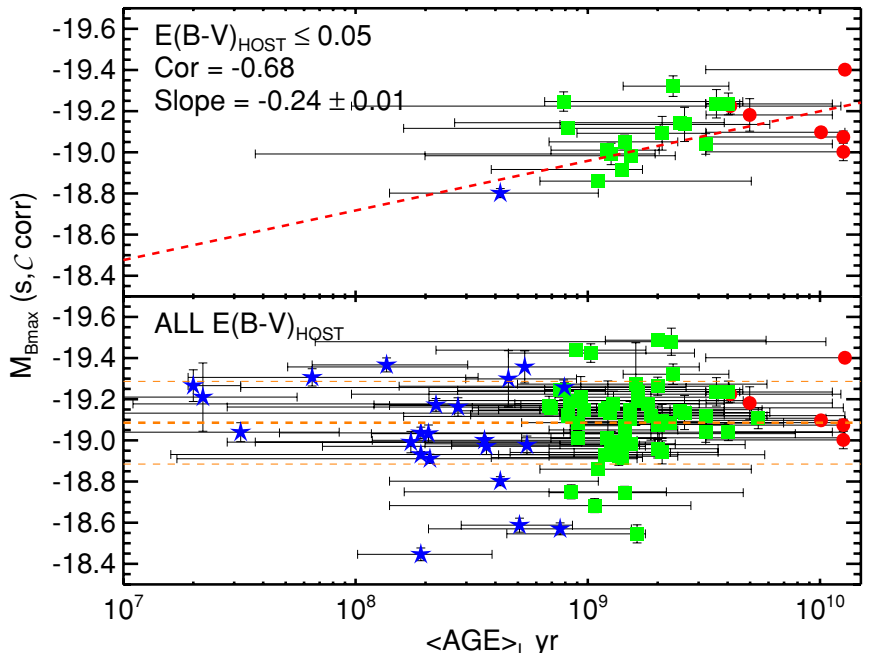

Figure 9. SN Ia stretch-color-corrected $M_{B}$ as a function of host luminosityweighted age for hosts in the Hubble flow $(z>0.013)$. The host sSFR is coded as in Figure 4. Since we are applying stretch and color corrections, we cut the sample to remove $\mathrm{SNe}$ with $s<0.75$ and $\mathcal{C}>0.7$. The bottom plot shows the Hubble flow subset $(N=85)$ with no cut on host extinction, while the top plot shows a low host extinction subset $(N=22)$ with $E(B-V)_{\text {HOST }} \leqslant 0.05$. We see no residual trend with host age for the larger set. The host extinction cut removes the youngest galaxies as expected. The residual trend in the low host extinction subset is indicated by the dashed line in the top plot. This trend has a significance of $2.1 \sigma$ when compared with a distribution of correlations created when drawing 22 data points at random from the total sample 10,000 times.

(A color version of this figure is available in the online journal.)

bottom panel plot shows no obvious residual trend with age for the larger sample. The residual trend apparent in the low host extinction subset has a correlation of -0.68 . To determine the significance of this correlation, we selected 22 hosts at random from the full set of 85 hosts 10,000 times, and ran the same linear regression analysis on each randomly selected sample. The low host extinction sample is a $2.1 \sigma$ outlier when compared with the resulting distribution of random correlations.

\subsection{Host Metallicity versus SN Ia ${ }^{56} \mathrm{Ni}$ Mass}

Here we complement the higher-redshift $(0.2<z<0.75)$ sample of H09 by using our nearby $(0.013<z<0.06)$ sample of SNe Ia. This local sample, in addition to testing the Timmes et al. (2003) model in the nearby universe, will provide a test of the method developed in $\mathrm{H} 09$ for deriving Ni masses and host metallicities.

\subsubsection{Host Metallicities from Host Masses}

H09 point out the difficulties in using spectroscopic line indices (Hamuy et al. 2000; Gallagher et al. 2008) or line ratios (Gallagher et al. 2005) to derive host metallicities to compare with SN Ia intrinsic luminosity or ${ }^{56} \mathrm{Ni}$ mass. Instead, they use the mass-metallicity relation of Tremonti et al. (2004). There are drawbacks to using this trend, which uses gas-phase metallicity, on all galaxies regardless of gas content. However, as pointed out in $\mathrm{H} 09$, there are good reasons for assuming this method is accurate enough for the purposes here (see H09, Section 3.2). We also repeat the caution from $\mathrm{H} 09$ that gas-phase metallicity is not the same as stellar metallicity, although they should be correlated (Fernandes et al. 2005). The apparent scatter in the Tremonti relationship is 0.1 dex, which we add in quadrature to our errors when we estimate host metallicities. For consistency,

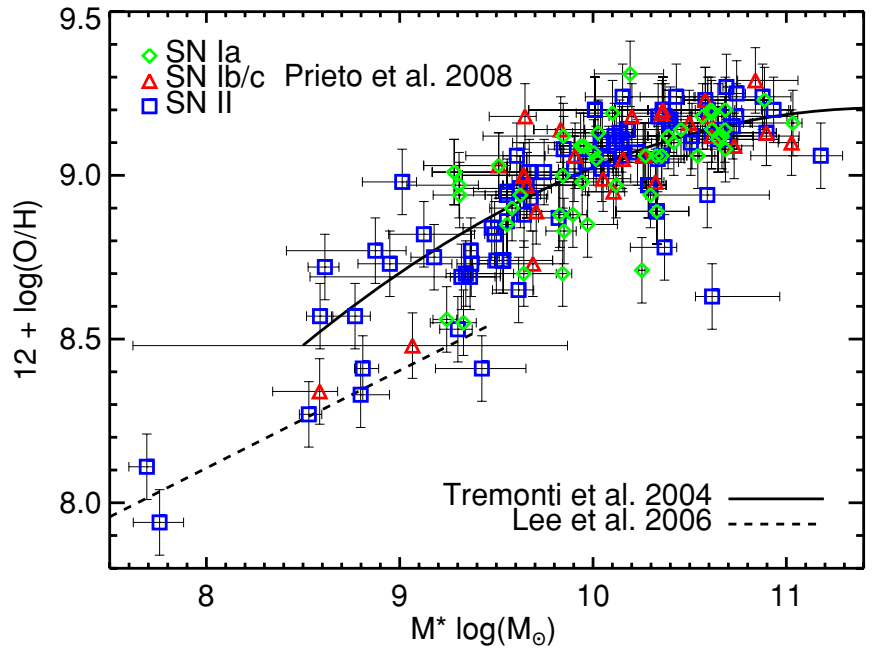

Figure 10. Metallicity plotted as a function of PEGASE.2 SED fitted stellar mass for the hosts from Prieto et al. (2008) for which we had sufficient integrated host photometry to derive good SED fits. The relation from Tremonti et al. (2004) is indicated as the solid thick line, and the low-metallicity extension from Lee et al. (2006) is indicated by the dashed line. Open diamonds indicate hosts of SNe Ia, open triangles indicate hosts of $\mathrm{SNe} \mathrm{Ib} / \mathrm{c}$, and open squares indicate hosts of SNe II. The Prieto sample is poorly sampled at masses below $\sim 10^{9.2} M_{\odot}$. This is most likely due to selection effects from host-targeted local SN surveys that rarely target low-mass hosts. Most of our local well fit SNe are in hosts with $M_{*} \gtrsim 10^{9} M_{\odot}$ (see Figure 6 ), and thus, we should not be overly influenced by this selection effect.

(A color version of this figure is available in the online journal.)

we use host masses derived using the same technique applied in H09 and outlined in Sullivan et al. (2006).

We test the use of the Tremonti et al. (2004) mass-metallicity relation for low- $z$ SN hosts by using the $\mathrm{SN}$ host metallicity data from Prieto et al. (2008). Their metallicities come from the SDSS as were those used in Tremonti et al. (2004). A comparison of the two results should reveal any biases in the low $z$ SN host sample. We have sufficiently good photometry for 170 SN hosts in the Prieto et al. (2008) catalog to perform this comparison. These data are also from the GLGA and will be published in M. Seibert et al. (2010, in preparation). We do not place any requirements on the SNe in this sample other than that they have metallicities from Prieto et al. (2008), and that we have enough host photometry for a good SED fit. Figure 10 plots our SED-fit stellar masses against the metallicities from Prieto et al. (2008). The relation from Tremonti et al. (2004) is shown as the solid line and the low-metallicity extension from Lee et al. (2006) is shown as the dashed line for comparison with the host data. The hosts with masses in the range $9<\log M_{*}<11$ appear to follow the Tremonti relation well, while hosts with $\log M_{*}<9$ appear to follow the Lee relation, albeit with a sparser sampling.

We will be looking for average trends in ${ }^{56} \mathrm{Ni}$ mass with metallicity, therefore we want to be sure to sample the range of the scatter in the Tremonti relation so we are not biased toward one side or the other by observational effects. The host mass range for our low- $z$ SNe Ia with well-fit light curves is primarily above $\log M_{*}=9$ (see Figure 6 ). The scatter in the massmetallicity relation for $\mathrm{SN}$ hosts appears to be well sampled above this mass; thus, we should be less subject to observational biases that could be in effect below this host mass. We also need not use the low-metallicity extension from Lee et al. (2006) for our base sample of SN Ia hosts. 


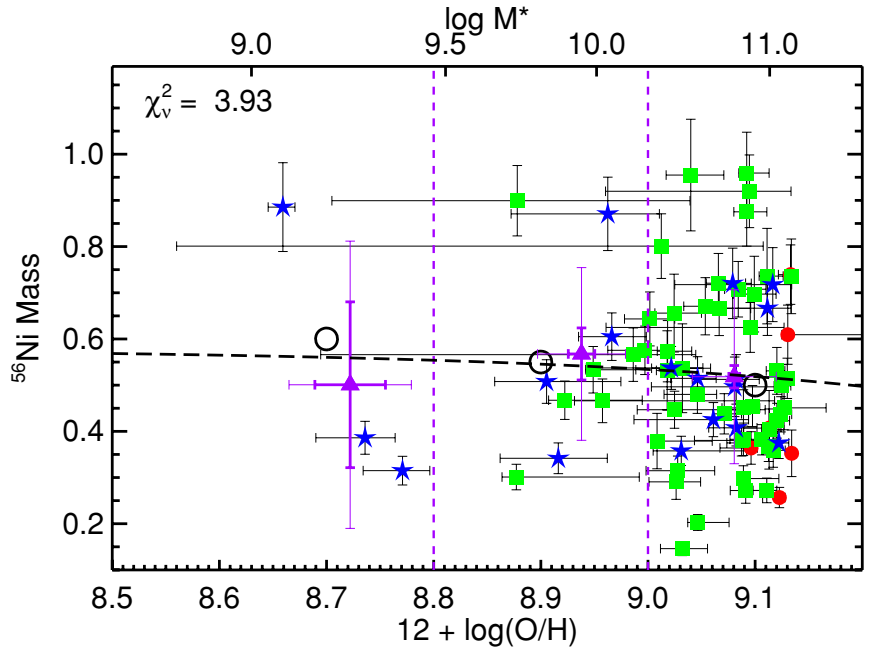

Figure 11. SN Ia ${ }^{56} \mathrm{Ni}$ mass as a function of metallicity. The host sSFR is coded as in Figure 4. The average ${ }^{56} \mathrm{Ni}$ masses in $(\mathrm{O} / \mathrm{H})$ bins of 0.2 dex starting at $12+\log (\mathrm{O} / \mathrm{H})=8.6$ (vertical dashed lines) are plotted as filled triangles. The thin error bars are the rms in each bin, while the thick error bars are the errors in the mean within the bin. The data from H09 are shown as the large open circles. The expected trend with metallicity from the Timmes et al. (2003) model, altered for thin disk $\mathrm{O} / \mathrm{Fe}$, is plotted as the thick dashed line. The average trend is consistent with that predicted by Timmes et al. (2003) as was found by H09 for an intermediate redshift sample of SNe Ia $(0.2<z<0.75)$.

(A color version of this figure is available in the online journal.)

\subsubsection{SN Ia ${ }^{56} \mathrm{Ni}$ Masses}

$\mathrm{H} 09$ present a technique for estimating the $\mathrm{SN}$ Ia ${ }^{56} \mathrm{Ni}$ mass using Arnett's Rule (Arnett 1979, 1982) to convert bolometric luminosity and SN Ia rise time to ${ }^{56} \mathrm{Ni}$ mass. We use the identical technique on the well-sampled light curves for our local $\mathrm{SNe}$ Ia. Because the estimate of the bolometric luminosity assumes the luminosity distance is accurate, we use only the $\mathrm{SNe}$ in the Hubble flow (see Table 1).

The lowest stretch SNe Ia are also excluded from this experiment because they produce inaccurate ${ }^{56} \mathrm{Ni}$ masses using our technique. As previously stated, low-luminosity SNe Ia deviate from the normal trend of luminosity versus light-curve shape (Garnavich et al. 2004; Taubenberger et al. 2008), thus the bolometric luminosity is inaccurate. The SN Ia spectral templates currently available are also not representative of SNe Ia with $s<0.75$ (Conley et al. 2008). A higher-order relation between light-curve shape and luminosity that properly accounts for low-luminosity SNe Ia and better spectral templates would allow us to use these $\mathrm{SNe}$ to test this model (S. GonzalezGaitan et al. 2010, in preparation). They will be quite useful because the strongest effect from metallicity should occur in the faintest $\mathrm{SNe}$. For our plots of Ni mass, we exclude objects with $s<0.75$. To preclude inaccuracies due to excessive extinction we include only $\mathrm{SNe}$ Ia with $\mathcal{C}<0.4$. The final sample of $74 \mathrm{SNe}$ that pass these cuts is presented in Table 3, where the host and the derived ${ }^{56} \mathrm{Ni}$ masses and host metallicities are listed.

Figure 11 shows the $\mathrm{SN}$ Ia ${ }^{56} \mathrm{Ni}$ mass as a function of host metallicity for our final sample. We use the same symbol coding as in Figure 4. We have taken the average Ni mass in three bins of metallicity starting at $12+\log (\mathrm{O} / \mathrm{H})=8.6$ and having a width of 0.2 and plot these as filled triangles. The model from Timmes et al. (2003) altered for thin-disk O/Fe (H09) is overplotted as a thick dashed line. The average trend seen in H09 is shown by the open circles. Although our data are statistically consistent

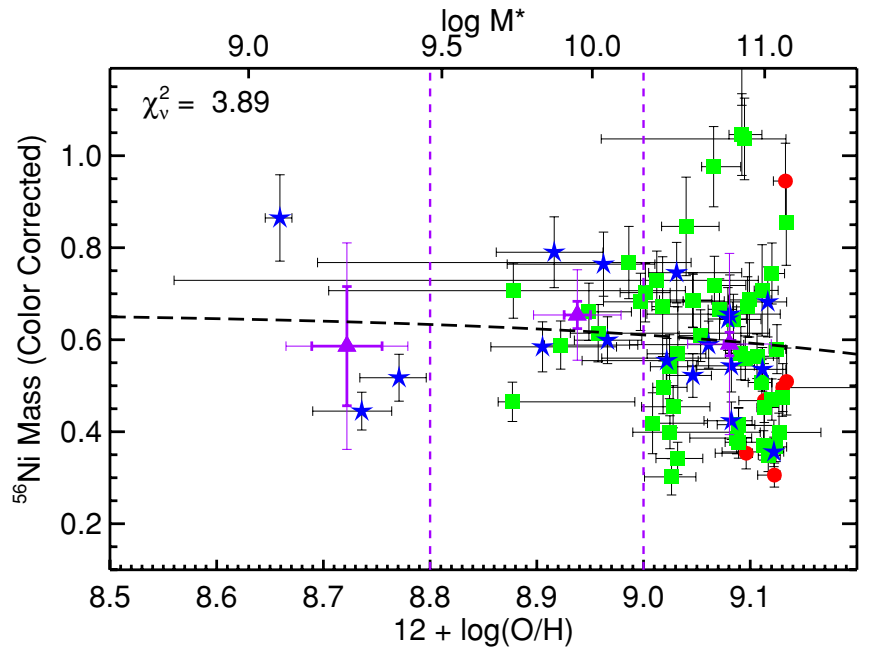

Figure 12. $\mathrm{SN}$ Ia ${ }^{56} \mathrm{Ni}$ mass as a function of metallicity with a correction for SN color applied (see the text). The host sSFR is coded as in Figure 4. The Timmes model is plotted as the thick dashed line. The color correction was not as effective as in the H09 sample and only reduces the $\chi_{v}^{2}$ from 3.93 to 3.89 , when comparing the data to the Timmes model.

(A color version of this figure is available in the online journal.)

with no trend, they are also consistent with the H09 trend and the Timmes model.

\subsubsection{Color Correction}

H09 apply a correction for the intrinsic SN Ia light-curve color to their derived $\mathrm{Ni}$ masses, after a color cut has been applied. Fainter SNe Ia are redder due to the intrinsic colorluminosity relation, or line-of-sight extinction, or both. Our $\mathrm{Ni}$ mass calculation requires the intrinsic luminosity, corrected for the dimming effects of dust, but not corrected for colorluminosity. Since the correction is based on the observed and not intrinsic color of the $\mathrm{SN}$, there is bound to be some error in assuming that there is no dust dimming (uncorrected $\mathrm{Ni}$ masses) or that the color-luminosity relation is purely due to dust (color corrected $\mathrm{Ni}$ masses). Both will be inaccurate at some level. One way to examine this is to calculate and compare the $\chi^{2} /$ dof or $\chi_{v}^{2}$ that results when comparing the corrected and uncorrected data to the Timmes model. When we apply the color correction, we find a mild improvement in the scatter as shown in Figure 12. When compared with the Timmes model, the $\chi_{v}^{2}$ is only marginally improved going from 3.93 (uncorrected) to 3.89 (color corrected).

These values of $\chi_{v}^{2}$ greater than one imply that our errors do not reflect the expected scatter of the data points around the Timmes model, assuming it is correct. H09 found that in order to achieve $\chi_{v}^{2}=1$, they had to add in quadrature an extra $0.16 M_{\odot}$ to their errors in Ni mass for their uncorrected masses. We also find that we need an extra error of $0.16 M_{\odot}$ in our uncorrected ${ }^{56} \mathrm{Ni}$ masses to achieve $\chi_{v}^{2}=1$. Another measure of effectiveness of the color correction is to see how much this extra error is reduced by the correction. For H09, it was significantly reduced from 0.16 (uncorrected) to 0.12 (color corrected) $M_{\odot}$. For our data there was only a marginal decrease in the extra scatter required for the color corrected ${ }^{56} \mathrm{Ni}$ masses $\left(0.162-0.158 M_{\odot}\right)$.

One possible explanation of this difference is the homogeneous light-curve photometry available for the H09 sample in contrast to the heterogeneous photometry available for our sample. The color cuts in $\mathrm{H} 09$ were made in a multicolor space that 
Table 3

${ }^{56} \mathrm{Ni}$ Mass and Host Metallicity

\begin{tabular}{|c|c|c|c|c|c|}
\hline $\mathrm{SN}$ & HOST & $\begin{array}{c}{ }^{56} \mathrm{Ni} \text { Mass } \\
\left(M_{\odot}\right)\end{array}$ & $\begin{array}{c}\text { Corrected } \\
{ }^{56} \mathrm{Ni} \text { Mass } \\
\left(M_{\odot}\right)\end{array}$ & $\begin{array}{c}\text { Mass-Metal } \\
12+\log (\mathrm{O} / \mathrm{H})\end{array}$ & $\begin{array}{c}\text { Prieto et al. } 2006 \\
12+\log (\mathrm{O} / \mathrm{H})\end{array}$ \\
\hline $1999 \mathrm{gd}$ & NGC 2623 & $0.15 \pm 0.02$ & $0.34 \pm 0.04$ & 9.10 & $\ldots$ \\
\hline $2006 \mathrm{cc}$ & UGC 10244 & $0.20 \pm 0.02$ & $0.68 \pm 0.06$ & 9.12 & $\ldots$ \\
\hline $2007 \mathrm{ci}$ & NGC 3873 & $0.26 \pm 0.02$ & $0.31 \pm 0.03$ & 9.19 & $\ldots$ \\
\hline $2001 N$ & NGC 3327 & $0.27 \pm 0.03$ & $0.57 \pm 0.06$ & 9.16 & $\ldots$ \\
\hline $2005 \mathrm{kc}$ & NGC 7311 & $0.27 \pm 0.03$ & $0.51 \pm 0.05$ & 9.18 & $\ldots$ \\
\hline 2001da & NGC 7780 & $0.29 \pm 0.04$ & $0.30 \pm 0.04$ & 9.10 & $\ldots$ \\
\hline $2006 \mathrm{bq}$ & NGC 6685 & $0.30 \pm 0.03$ & $0.41 \pm 0.04$ & 9.16 & $\ldots$ \\
\hline $2006 \mathrm{ar}$ & $M+11-13-36$ & $0.30 \pm 0.03$ & $0.47 \pm 0.04$ & 8.95 & 9.09 \\
\hline $2004 \mathrm{~L}$ & $\mathrm{M}+03-27-38$ & $0.31 \pm 0.03$ & $0.45 \pm 0.05$ & 9.10 & $\ldots$ \\
\hline $2007 \mathrm{bz}$ & IC3918 & $0.31 \pm 0.03$ & $0.52 \pm 0.05$ & 8.84 & $\ldots$ \\
\hline 2005ls & $\mathrm{M}+07-07-01$ & $0.34 \pm 0.03$ & $0.79 \pm 0.08$ & 8.99 & $\ldots$ \\
\hline 2008af & UGC 9640 & $0.35 \pm 0.05$ & $0.51 \pm 0.07$ & 9.21 & $\ldots$ \\
\hline 1996bo & NGC 673 & $0.36 \pm 0.03$ & $0.75 \pm 0.07$ & 9.10 & $\cdots$ \\
\hline $1994 \mathrm{M}$ & NGC 4493 & $0.36 \pm 0.04$ & $0.35 \pm 0.04$ & 9.19 & $\cdots$ \\
\hline $2006 \mathrm{~N}$ & $M+11-08-12$ & $0.36 \pm 0.04$ & $0.35 \pm 0.03$ & 9.17 & $\ldots$ \\
\hline $1999 \mathrm{cc}$ & NGC 6038 & $0.37 \pm 0.03$ & $0.37 \pm 0.03$ & 9.18 & $\ldots$ \\
\hline $2002 \mathrm{he}$ & UGC 4322 & $0.37 \pm 0.03$ & $0.36 \pm 0.03$ & 9.19 & $\ldots$ \\
\hline 2006al & A103929+0511 & $0.38 \pm 0.06$ & $0.42 \pm 0.07$ & 9.08 & $\ldots$ \\
\hline $2003 \mathrm{U}$ & NGC 6365A & $0.38 \pm 0.03$ & $0.39 \pm 0.03$ & 9.16 & $\ldots$ \\
\hline $2006 \mathrm{ac}$ & NGC 4619 & $0.38 \pm 0.03$ & $0.56 \pm 0.05$ & 9.18 & $\ldots$ \\
\hline $2007 b c$ & UGC 6332 & $0.38 \pm 0.04$ & $0.38 \pm 0.03$ & 9.16 & $\ldots$ \\
\hline 2004as & A $112539+2249$ & $0.39 \pm 0.04$ & $0.44 \pm 0.04$ & 8.81 & $\ldots$ \\
\hline $2001 \mathrm{ie}$ & UGC 5542 & $0.40 \pm 0.09$ & $0.45 \pm 0.10$ & 9.19 & $\ldots$ \\
\hline $2006 \mathrm{ej}$ & NGC 191A & $0.41 \pm 0.04$ & $0.47 \pm 0.05$ & 9.19 & $\ldots$ \\
\hline $2006 \mathrm{sr}$ & UGC 14 & $0.41 \pm 0.04$ & $0.42 \pm 0.04$ & 9.15 & $\cdots$ \\
\hline $2006 \mathrm{bt}$ & $M+03-41-04$ & $0.42 \pm 0.04$ & $0.74 \pm 0.07$ & 9.19 & $\cdots$ \\
\hline $2003 W$ & UGC 5234 & $0.43 \pm 0.04$ & $0.59 \pm 0.05$ & 9.13 & $\ldots$ \\
\hline $2005 \mathrm{ki}$ & NGC 3332 & $0.44 \pm 0.04$ & $0.37 \pm 0.03$ & 9.20 & $\ldots$ \\
\hline $2002 \mathrm{bf}$ & CGCG266-031 & $0.44 \pm 0.04$ & $0.67 \pm 0.07$ & 9.14 & $\ldots$ \\
\hline $2005 \mathrm{iq}$ & M-03-01-08 & $0.45 \pm 0.04$ & $0.40 \pm 0.04$ & 9.10 & $\ldots$ \\
\hline $2007 \mathrm{bd}$ & UGC 4455 & $0.45 \pm 0.04$ & $0.42 \pm 0.04$ & 9.16 & $\ldots$ \\
\hline 1992bl & E291-G11 & $0.45 \pm 0.05$ & $0.40 \pm 0.04$ & 9.20 & $\ldots$ \\
\hline $2002 \mathrm{de}$ & NGC 6104 & $0.45 \pm 0.04$ & $0.67 \pm 0.07$ & 9.17 & $\ldots$ \\
\hline 1992ag & E508-G67 & $0.47 \pm 0.05$ & $0.61 \pm 0.06$ & 9.03 & $\ldots$ \\
\hline $2006 \mathrm{cp}$ & UGC 7357 & $0.47 \pm 0.04$ & $0.59 \pm 0.05$ & 8.99 & $\ldots$ \\
\hline $2006 \mathrm{~S}$ & UGC 7934 & $0.48 \pm 0.04$ & $0.69 \pm 0.06$ & 9.12 & $\ldots$ \\
\hline 2006en & $M+05-54-41$ & $0.50 \pm 0.06$ & $0.66 \pm 0.09$ & 9.15 & $\cdots$ \\
\hline 2004eo & NGC 6928 & $0.50 \pm 0.05$ & $0.58 \pm 0.05$ & 9.20 & $\cdots$ \\
\hline $2000 \mathrm{fa}$ & UGC 3770 & $0.51 \pm 0.05$ & $0.58 \pm 0.05$ & 8.98 & $\ldots$ \\
\hline 20070 & UGC 9612 & $0.51 \pm 0.05$ & $0.54 \pm 0.06$ & 9.15 & $\ldots$ \\
\hline $2002 \mathrm{jy}$ & NGC 477 & $0.51 \pm 0.05$ & $0.52 \pm 0.05$ & 9.12 & $\ldots$ \\
\hline $2006 a z$ & NGC 4172 & $0.51 \pm 0.04$ & $0.47 \pm 0.04$ & 9.20 & $\ldots$ \\
\hline $2002 \mathrm{ha}$ & NGC 6962 & $0.53 \pm 0.05$ & $0.47 \pm 0.04$ & 9.19 & 8.94 \\
\hline 2006on & A215558-0104 & $0.53 \pm 0.09$ & $0.67 \pm 0.11$ & 9.09 & $\ldots$ \\
\hline $1996 \mathrm{C}$ & $M+08-25-47$ & $0.53 \pm 0.05$ & $0.66 \pm 0.06$ & 9.02 & $\ldots$ \\
\hline 2001en & NGC 523 & $0.54 \pm 0.10$ & $0.57 \pm 0.10$ & 9.10 & $\ldots$ \\
\hline $2005 \mathrm{~ms}$ & UGC 4614 & $0.54 \pm 0.05$ & $0.55 \pm 0.05$ & 9.09 & 9.06 \\
\hline 1996bv & UGC 3432 & $0.57 \pm 0.06$ & $0.77 \pm 0.08$ & 9.06 & $\ldots$ \\
\hline 2006te & A $081144+4133$ & $0.57 \pm 0.07$ & $0.50 \pm 0.06$ & 9.09 & 9.10 \\
\hline $1999 \mathrm{dk}$ & UGC 1087 & $0.58 \pm 0.05$ & $0.68 \pm 0.06$ & 9.07 & $\ldots$ \\
\hline $2007 \mathrm{~F}$ & UGC 8162 & $0.60 \pm 0.05$ & $0.60 \pm 0.05$ & 9.04 & 9.05 \\
\hline $1998 d x$ & UGC 11149 & $0.61 \pm 0.06$ & $0.50 \pm 0.05$ & 9.20 & $\ldots$ \\
\hline $2006 a x$ & NGC 3663 & $0.62 \pm 0.05$ & $0.56 \pm 0.05$ & 9.17 & $\ldots$ \\
\hline $2001 \mathrm{fe}$ & UGC 5129 & $0.64 \pm 0.06$ & $0.70 \pm 0.06$ & 9.07 & $\ldots$ \\
\hline 1992P & IC3690 & $0.66 \pm 0.08$ & $0.54 \pm 0.07$ & 9.10 & $\ldots$ \\
\hline $1998 \mathrm{ab}$ & NGC 4704 & $0.67 \pm 0.06$ & $0.72 \pm 0.06$ & 9.14 & 9.16 \\
\hline $2001 \mathrm{ba}$ & M-05-28-01 & $0.67 \pm 0.06$ & $0.54 \pm 0.05$ & 9.18 & $\ldots$ \\
\hline $1994 \mathrm{~S}$ & NGC 4495 & $0.67 \pm 0.06$ & $0.61 \pm 0.06$ & 9.13 & $\ldots$ \\
\hline $1998 \mathrm{~V}$ & NGC 6627 & $0.70 \pm 0.08$ & $0.69 \pm 0.08$ & 9.17 & $\ldots$ \\
\hline 1999aa & NGC 2595 & $0.71 \pm 0.06$ & $0.64 \pm 0.05$ & 9.16 & $\ldots$ \\
\hline $1991 \mathrm{U}$ & IC4232 & $0.72 \pm 0.08$ & $0.68 \pm 0.08$ & 9.19 & $\cdots$ \\
\hline $2001 \mathrm{az}$ & UGC 10483 & $0.72 \pm 0.08$ & $0.65 \pm 0.07$ & 9.15 & $\cdots$ \\
\hline $2005 \mathrm{eq}$ & M-01-09-06 & $0.72 \pm 0.06$ & $0.98 \pm 0.09$ & 9.14 & $\cdots$ \\
\hline $2007 \mathrm{ae}$ & UGC 10704 & $0.74 \pm 0.08$ & $0.86 \pm 0.09$ & 9.21 & $\ldots$ \\
\hline
\end{tabular}


Table 3

(Continued)

\begin{tabular}{lccccc}
\hline \hline SN & HOST & $\begin{array}{c}56 \text { Ni Mass } \\
\left(M_{\odot}\right)\end{array}$ & $\begin{array}{c}\text { Corrected } \\
56 \text { Ni Mass } \\
\left(M_{\odot}\right)\end{array}$ & $\begin{array}{c}\text { Mass-Metal } \\
12+\log (\mathrm{O} / \mathrm{H})\end{array}$ & $\begin{array}{c}\text { Prieto et al. 2006 } \\
12+\log (\mathrm{O} / \mathrm{H})\end{array}$ \\
\hline $2002 \mathrm{ck}$ & UGC 10030 & $0.74 \pm 0.10$ & $0.71 \pm 0.10$ & 9.18 & 9.23 \\
$2008 \mathrm{bf}$ & NGC 4055 & $0.74 \pm 0.06$ & $0.94 \pm 0.08$ & 9.21 & $\ldots$ \\
$2002 \mathrm{hu}$ & M+06-06-12 & $0.80 \pm 0.07$ & $0.73 \pm 0.06$ & 9.08 & $\ldots$ \\
$2000 \mathrm{ca}$ & E383-G32 & $0.87 \pm 0.08$ & $0.76 \pm 0.07$ & 9.03 & $\ldots$ \\
$2001 \mathrm{~V}$ & NGC 3987 & $0.88 \pm 0.07$ & $1.05 \pm 0.09$ & 9.16 & $\ldots$ \\
$1991 \mathrm{ag}$ & IC4919 & $0.89 \pm 0.10$ & $0.86 \pm 0.09$ & 8.73 & $\ldots$ \\
$1992 \mathrm{bc}$ & E300-G09 & $0.90 \pm 0.08$ & $0.71 \pm 0.06$ & 8.95 & $\ldots$ \\
$2006 \mathrm{fa}$ & M+07-36-33 & $0.92 \pm 0.08$ & $1.04 \pm 0.09$ & 9.17 & $\ldots$ \\
$1999 \mathrm{dq}$ & A125924+2820 & $0.95 \pm 0.12$ & $0.85 \pm 0.11$ & 9.11 & $\ldots$ \\
\hline
\end{tabular}

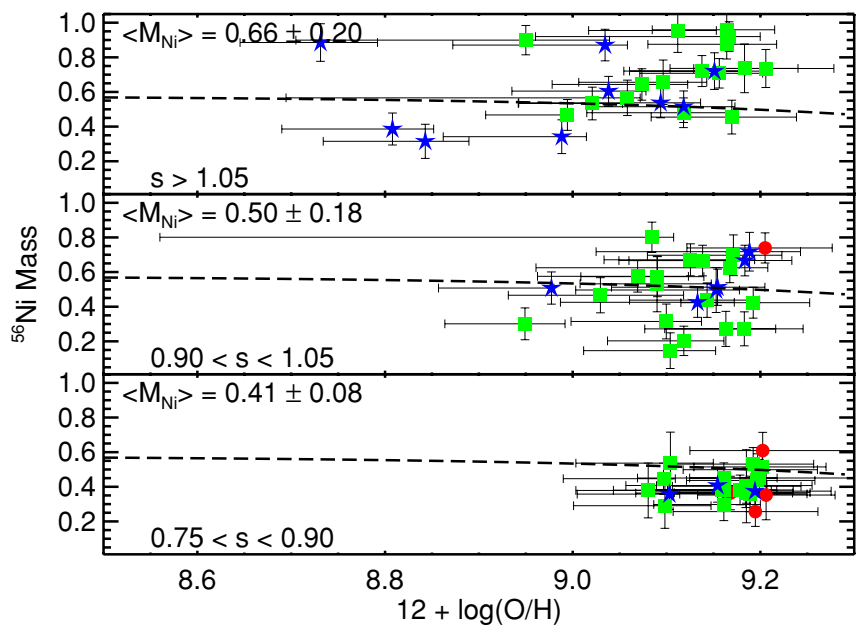

Figure 13. SN Ia ${ }^{56} \mathrm{Ni}$ mass (uncorrected) as a function of metallicity for three stretch bins, as annotated. The host sSFR is coded as in Figure 4. The Timmes model is plotted as the thick dashed line. The average ${ }^{56} \mathrm{Ni}$ mass increases with stretch across the three bins, as expected. The range of host metallicity and the scatter in Ni mass decreases toward lower stretch as was seen in the higher-redshift sample from H09.

(A color version of this figure is available in the online journal.)

included wavelength regions that were not available for all the $\mathrm{SNe}$ in the local sample (see their Section 3.3). Consequently, our color cut here was in a single color $(B-V)$ and may not have been as effective at removing outliers in the color corrected ${ }^{56} \mathrm{Ni}$ mass. We point out that there is an obvious reduction by the color correction in the scatter of $\mathrm{Ni}$ mass for lower-metallicity hosts, and the biggest outliers in the color-corrected plot (Figure 12) are near the highest metallicity. The fact that the color correction provides any improvement at all is consistent with the idea that at least some part of the color-luminosity relation is due to host dust extinction.

\subsubsection{SN Ia ${ }^{56}$ Ni Mass in Three Stretch Bins}

Figure 13 shows the uncorrected Ni mass versus metallicity divided in three stretch bins (see H09, Figure 10). We do not sample as large a range of metallicity as H09; nonetheless, we do see similar features. The range of host metallicity for the higherstretch $\mathrm{SNe}$ Ia is roughly double that for the lower-stretch group. We expect the average ${ }^{56} \mathrm{Ni}$ mass to increase with stretch due to stretch-luminosity, but we also see that the scatter in the masses is lowest in the lowest stretch group. The stretch cut in the low-stretch bin undoubtedly limits the Ni mass in this bin

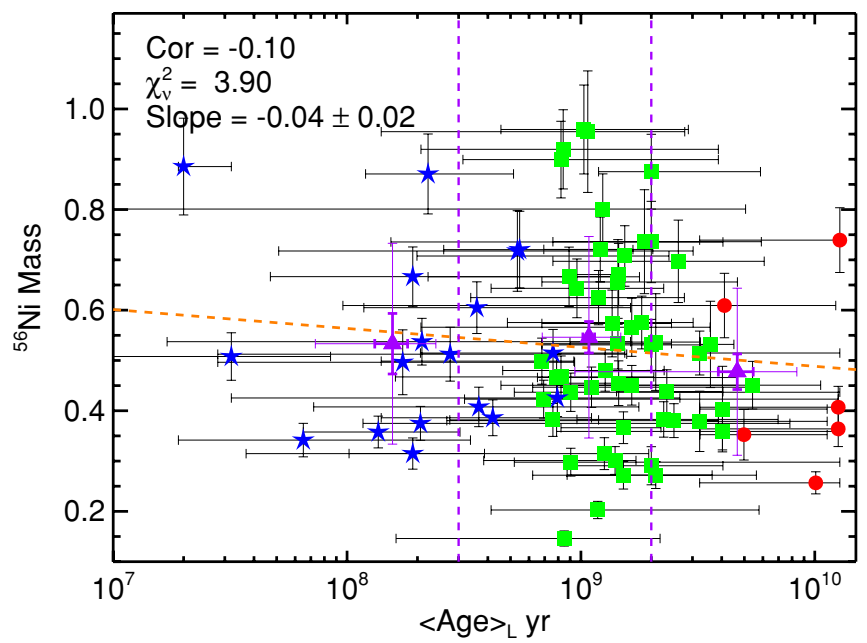

Figure 14. SN Ia ${ }^{56} \mathrm{Ni}$ mass, with no color correction, as a function of luminosityweighted host age. The host sSFR is coded as in Figure 4. The average ${ }^{56} \mathrm{Ni}$ mass in three bins dividing the sample at luminosity-weighted ages of $3 \times 10^{8}$ and $2 \times 10^{9} \mathrm{yr}$ (vertical dashed lines) is plotted as filled triangles. The thin error bars are the rms in each bin while the thick error bars are the error in the mean in each bin. A linear fit is shown as the dashed line and the results of the correlation are annoted on the plot. The correlation is weaker and the slope is shallower than what was seen in the H09 sample (see their Figure 8). The linear trend with age produces a $\chi_{v}^{2}$ similar to the Timmes model. Combined with H09, Figure 8 , we note that SNe Ia with ${ }^{56} \mathrm{Ni}$ masses greater than $\sim 0.5 M_{\odot}$ are rare in hosts with luminosity-weighted ages greater than $\sim 3 \times 10^{9} \mathrm{yr}$.

(A color version of this figure is available in the online journal.)

(see H09, Section 4.5), but at the low Ni mass end our local sample should be less subject to Malquist biases. Another effect is the color cut of $\mathcal{C}<0.4$, which precludes the reddest, faintest, and therefore lowest $\mathrm{Ni}$ mass SNe. Nonetheless, the trends are suggestive and support the idea that lower-stretch SNe Ia appear in higher-metallicity hosts.

\subsection{Host Age versus SN Ia ${ }^{56} \mathrm{Ni}$ Mass}

Due to the age-metallicity degeneracy, we present plots of ${ }^{56} \mathrm{Ni}$ mass against luminosity-weighted age for the uncorrected (Figure 14) and color-corrected (Figure 15) Ni masses. We use the same symbol scheme as previous plots. We use a linear regression analysis to measure and test the correlation between age and Ni mass. The color correction slightly steepens the slope of the fit (from $-0.04 \pm 0.02$ to $-0.06 \pm 0.02$ ), and does improve the correlation from -0.10 (uncorrected) to -0.16 (color corrected) 


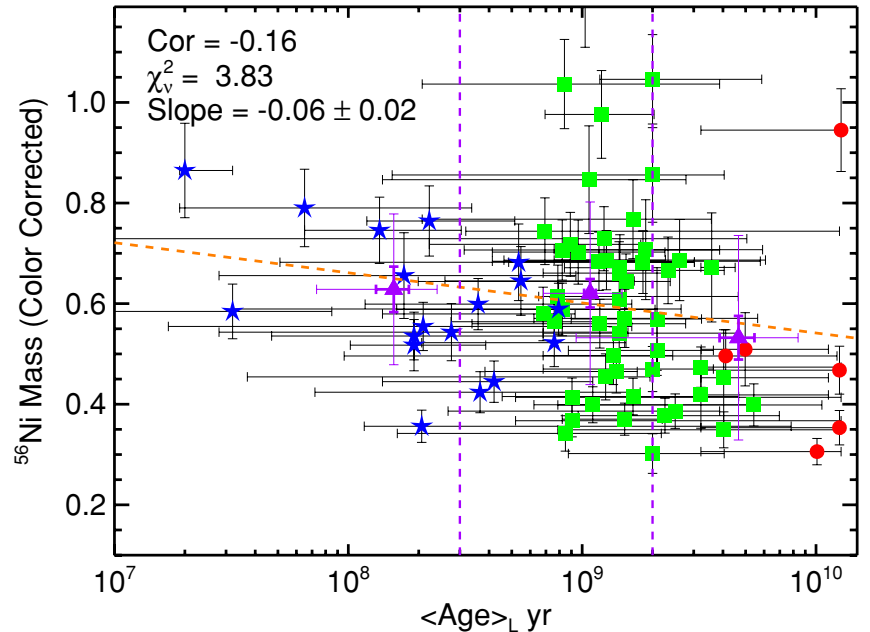

Figure 15. SN Ia ${ }^{56} \mathrm{Ni}$ mass, corrected for $\mathrm{SN}$ color, as a function of luminosityweighted host age. The host sSFR is coded as in Figure 4, and the bins for the averages are the same as in Figure 14. The color correction increases the correlation and marginally changes the slope. The rarity of higher $\mathrm{Ni}$ mass $\mathrm{SNe}$ Ia in the oldest hosts is still apparent (see Figure 14, and H09, Figure 9).

(A color version of this figure is available in the online journal.)

The age-Ni-mass slopes reported in H09 for their sample are significantly steeper: $-0.15 \pm 0.03$ (uncorrected) and $-0.11 \pm$ 0.03 (color corrected). The correlations are also larger: -0.38 (uncorrected) and -0.37 (color corrected). Examining Figures 8 and 9 in $\mathrm{H} 09$, we see that a large part of the correlation seems to originate in hosts with luminosity-weighted ages beyond $3 \mathrm{Gyr}$ which seem incapable of producing SNe with ${ }^{56} \mathrm{Ni}$ masses above $0.5 M_{\odot}$ in the uncorrected plot (their Figure 8) and above $0.6 M_{\odot}$ in the corrected plot (their Figure 9). Below 3 Gyr, there appears to be very little correlation between age and Ni mass. We see a similar feature in our data as well (Figures 14 and 15), although not quite as strong. This feature implies a threshold that is reached at a luminosity-weighted age of $\sim 3 \mathrm{Gyr}$, rather than a uniform trend with age.

\section{DISCUSSION}

The implications of these results must be tempered by the limitations of the techniques. In particular, the SED-fitting method employed was optimized for higher-redshift galaxies, and we do not employ infrared data to constrain the SEDs of our low-redshift hosts. Our aim is to compare hosts at high and low redshifts using the same methods. We focus here on trends and this comparison, and will pursue more precise determinations of host parameters with infrared data and more detailed methods in a future paper.

We reiterate that the ages estimated with the PEGASE.2 fits are luminosity weighted and the masses are current stellar masses. The SFRs only measure current activity based on UV and optical data, and have not been modified by FIR data to account for dust. The global host extinctions are derived by extincting the entire SED and do not account for variable thermal components in the IR.

\subsection{Host Properties at High and Low Redshift}

Our results show features similar to those presented in higherredshift host studies using the same methods (Sullivan et al. 2006; Sullivan 2009; H09). Low-stretch SNe Ia appear to be rare in high sSFR hosts, and the average stretch of SNe Ia in the lowest sSFR hosts is smaller than in hosts with higher sSFR (see Figure 4). The average stretch of $\mathrm{SNe}$ in hosts younger than $\sim 2$ Gyr is close to 1 and for older hosts the average stretch appears to drop below 1 (see Figure 5). H09 were the first to plot host stellar mass against stretch, but their sample does not include SNe Ia with $s<0.75$ (see their Figure 4). The interesting feature in our plot (Figure 6) is the limited mass range for lowerstretch SNe. This feature is present in the H09 figure, but it is less pronounced due to having fewer low-stretch SNe in their sample.

The apparent stellar mass threshold of $10^{10} M_{\odot}$ for the hosts of low-luminosity $\mathrm{SNe}$ Ia is fairly robust against selection effects. Our sample includes many lower-mass hosts within which it is easier to detect low-luminosity SNe. That these SNe prefer higher-mass hosts supports the idea that they arise from an older progenitor population. Figures 4-6 show that the one high sSFR host (E445-G66) of a SN Ia with $s<0.80$ (SN1993H) is also massive, implying that the recently formed populations are mixed with the older populations that could be responsible for the low-stretch SN Ia. The correlation between mass and metallicity implies that metallicity could play a role as well (see Section 3.4). We see no low-mass, high sSFR hosts of low-stretch SNe Ia, and we also see few older, higher-mass hosts of the highest stretch SNe Ia. A method that can more accurately asses the star formation history of these hosts and the relative contributions from older and younger populations will produce a more definitive constraint on the progenitors of the low-luminosity SNe Ia (Schawinski 2009).

Our plot of SN peak color versus host age illustrates the difficulty of disentangling the source of the color (see Figure 7) since it shows no correlation. We are also challenged in our comparison with higher-redshift samples by the bias against SNe with very red peak colors intrinsic to cosmology surveys (see Section 2.1). Even in the local universe, our sample of six SNe Ia with $\mathcal{C}>0.7$ is too small for definitive interpretation. We point out one common feature of our figure and Figure 3 in Sullivan et al. (2009): very red SNe appear to be rare in hosts less than $0.5 \mathrm{Gyr}$ in luminosity-weighted age.

Based on Figure 11, we conclude that the model of Timmes et al. (2003), relating metallicity to produced ${ }^{56} \mathrm{Ni}$ mass, appears consistent with the derived host metallicities and SN Ia ${ }^{56} \mathrm{Ni}$ masses for $\mathrm{SNe}$ in the local universe as well as at higher redshifts (H09), although the local data are also consistent with no trend in these properties. The degeneracy between age and metallicity makes it difficult to decide which is the more important factor in determining the ${ }^{56} \mathrm{Ni}$ mass of hosted SNe Ia. The apparent transition at a luminosity-weighted host age of 3 Gyr (see Figure 14, and H09, Figure 8) could place interesting constraints on progenitor scenarios given that this implies a fairly long delay time for low ${ }^{56} \mathrm{Ni}$ mass, hence low-luminosity and low-stretch, SNe Ia.

\subsection{Host Properties and Cosmological Fitting}

It is encouraging that the features in plots of light-curve properties against host properties in local hosts are similar when compared with the SNLS sample out to $z=0.75$ (Astier et al. 2006; Howell et al. 2007; Sullivan et al. 2009), although a detailed comparison using Hubble diagram residuals will be needed to determine if any significant evolution of the population can be detected (A. Conley et al. 2010, in preparation; Sullivan et al. 2009). These trends do signal potential systematics for SN cosmology: as we extend the samples beyond redshift $z=0.75$, we know the mix of host populations will move toward lower-mass, higher sSFR galaxies. 
Our examination of low-extinction hosts suggests a trend in brightness with host age such that SNe Ia in older hosts appear brighter after correcting for stretch and color (see Figure 9). While the correlation we see is hardly significant $(2.1 \sigma)$, it does suggest that luminosity-weighted host age might be an interesting parameter to explore in cosmological fits of SNe Ia. A larger sample of SNe Ia from low-extinction hosts would allow a more definitive test of this correlation.

While there is no apparent trend in a similar plot in Gallagher et al. (2008, see their Figure 9), we see a trend that is marginally significant. There are several possible explanations for this difference. Since the hosts in the Gallagher et al. (2008) sample were selected morphologically, their sample may not be as free from dust extinction as originally thought. In addition, their corrections for light-curve shape are derived with a method that assumes a Milky-Way-like dust is responsible for residual color after a quadratic color-decline relation is subtracted (see comparison of fitting methods in Conley et al. 2008), which may not be appropriate for these hosts. The trend we see in Figure 9 is in the right sense to be caused by residual line-ofsight extinction since we would expect the SNe hosted by the oldest galaxies to have the least non-local extinction.

These data suggest that either the SNe Ia or the dust produced in galaxies of different host properties are different or both. Hicken et al. (2009b) showed a weak trend in the Hubble residuals when using samples divided into three host morphology bins, after removing the reddest $\mathrm{SNe}$. An exploration based on more physical host properties could provide a more accurate assessment of this effect (Sullivan et al. 2009). Further progress may be made by extending host photometry farther into the infrared to help constrain the host dust content and by more detailed fitting of host star formation history to constrain the most recent episode of star formation (e.g., Schawinski 2009).

\section{SUMMARY}

We gathered the host integrated photometry for 168 wellobserved SNe Ia and correlated the derived host properties with the hosted SN Ia light-curve properties. We used the derived average host extinction to isolate a set of SNe Ia arising in hosts with low extinction. We used the methods outlined in H09 to compare host metallicity with $\mathrm{SN}$ Ia ${ }^{56} \mathrm{Ni}$ mass.

With our low-redshift sample, we have corroborated trends in SN Ia light-curve shape with host properties observed in higherredshift SN Ia samples of similar size (Sullivan et al. 2006). Specifically, we find that the higher the sSFR of the host galaxy, the brighter and slower the SNe Ia that are produced, on average. We find that the typical stretch of the SNe Ia produced begins to drop after the host galaxy reaches a luminosity-weighted age of 1 Gyr. We find that low-stretch $(s<0.80)$ SNe Ia in the local universe prefer hosts with stellar masses above $10^{10} M_{\odot}$. This is unlikely to be due to a Malmquist bias, since lower-luminosity $\mathrm{SNe}$ are easier to detect against lower-mass, lower-luminosity hosts. We find that fainter, faster SNe Ia do indeed prefer more massive and presumably higher-metallicity hosts, supporting the link between the production of ${ }^{56} \mathrm{Ni}$ and progenitor metallicity.

We suggest that it is likely that SN Ia cosmological fitting could be improved by adding a parameter characterizing the host properties to account for the impact of galaxy evolution on the evolution of SNe Ia with redshift. The trends in host properties with SN Ia light-curve properties support this idea and suggest that perhaps host age would be a good property to use.

Our sample shows no obvious trend in host properties with SN Ia peak color, except that all the reddest SNe Ia appear in hosts with ages around 1 Gyr. We find that the reddest SNe Ia appear in hosts with a range of derived host extinctions.

Our low host extinction sample suggests a residual trend of SN peak absolute brightness and host age such that SNe Ia in older hosts appear brighter after their peak brightnesses are corrected for light-curve shape and color (i.e., stretch-color corrected). One possible explanation for this is that the color correction leaves a small residual brightness trend that is due to extinction and that older hosts contain less dust than younger hosts. This residual trend is consistent with the one reported in Hicken et al. (2009b), who find that SNe Ia in early-type galaxies are brighter, after shape and color correction, than SNe Ia appearing in late-type hosts.

For SNe Ia with $s>0.75$ and $\mathcal{C}<0.4$, we find local SNe Ia host metallicities and $\mathrm{SN}$ Ia ${ }^{56} \mathrm{Ni}$ masses are consistent with the model of Timmes et al. (2003) altered for thin-disk O/Fe as in $\mathrm{H} 09$, but with an additional scatter of $0.16 M_{\odot}$. The local data are also consistent with no trend. The failure of the SN color correction to significantly reduce the scatter in the Ni masses is likely due to the heterogeneous photometry of the local SNe Ia. In spite of this, the trend of average ${ }^{56} \mathrm{Ni}$ mass and the scatter with stretch is very similar to that seen in H09.

We examined the trend in ${ }^{56} \mathrm{Ni}$ mass with host luminosityweighted age and found a shallower slope and a weaker correlation than that shown in $\mathrm{H} 09$ for a higher-redshift sample $(0.2<z<0.75)$. We point out what appears to be a threshold luminosity-weighted age of 3 Gyr apparent in our sample and the H09 sample, above which a host is less likely to produce $\mathrm{SNe}$ Ia with ${ }^{56} \mathrm{Ni}$ masses greater than $\sim 0.5 M_{\odot}$. Below this age threshold, there appears to be little correlation between ${ }^{56} \mathrm{Ni}$ mass and host age.

J.N. would like to thank Fillipo Mannucci, Dan Maoz, Massimo Della Valle, and Patrizia Braschi, the organizers of the 2008 May SN Ia rates conference in Florence, Italy where a preliminary version of this work was presented and discussed. We acknowledge the useful comments by the anonymous referee.

GALEX (Galaxy Evolution Explorer) is a NASA Small Explorer, launched in 2003 April. We gratefully acknowledge NASA's support for construction, operation, and science analysis for the GALEX mission, developed in cooperation with the Centre National d'Etudes Spatiales of France and the Korean Ministry of Science and Technology.

This research has made use of the NASA/IPAC Extragalactic Database (NED), which is operated by the Jet Propulsion Laboratory, California Institute of Technology, under contract with the National Aeronautics and Space Administration.

Funding for the SDSS and SDSS-II has been provided by the Alfred P. Sloan Foundation, the Participating Institutions, the National Science Foundation, the U.S. Department of Energy, the National Aeronautics and Space Administration, the Japanese Monbukagakusho, the Max Planck Society, and the Higher Education Funding Council for England. The SDSS Web Site is http://www.sdss.org/.

The SDSS is managed by the Astrophysical Research Consortium for the Participating Institutions. The Participating Institutions are the American Museum of Natural History, Astrophysical Institute Potsdam, University of Basel, University of Cambridge, Case Western Reserve University, University of Chicago, Drexel University, Fermilab, the Institute for Advanced Study, the Japan Participation Group, Johns Hopkins University, the Joint Institute for Nuclear Astrophysics, the 
Kavli Institute for Particle Astrophysics and Cosmology, the Korean Scientist Group, the Chinese Academy of Sciences (LAMOST), Los Alamos National Laboratory, the Max-Planck Institute for Astronomy (MPIA), the Max-Planck Institute for Astrophysics (MPA), New Mexico State University, Ohio State University, University of Pittsburgh, University of Portsmouth, Princeton University, the United States Naval Observatory, and the University of Washington.

\section{REFERENCES}

Arnett, W. D. 1979, ApJ, 230, L37

Arnett, W. D. 1982, ApJ, 253, 785

Astier, P., et al. 2006, A\&A, 447, 31

Bronder, T. J., et al. 2008, A\&A, 477, 717

Calzetti, D., Kinney, A. L., \& Storchi-Bergmann, T. 1994, ApJ, 429, 582

Colgate, S. A., \& McKee, C. 1969, ApJ, 157, 623

Conley, A., Carlberg, R. G., Guy, J., Howell, D. A., Jha, S., Riess, A. G., \& Sullivan, M. 2007, ApJ, 664, L13

Conley, A. J., et al. 2008, ApJ, 681, 482

de Vaucouleurs, G. 1959, in Handbuch der Physik, Vol. 53, ed. E. Flugge (Berlin: Springer-Verlag), 275

de Vaucouleurs, G., de Vaucouleurs, A., Corwin, H. G., Buta, R. J., Paturel, G., \& Fouque, P. 1991, Third Reference Catalogue of Bright Galaxies, Vols. 1-3 (Berlin: Springer-Verlag)

Fernandes, R. C., Mateus, A., Sodré, L., Stasińska, G., \& Gomes, J. M. 2005, MNRAS, 358, 363

Fioc, M., \& Rocca-Volmerange, B. 1997, A\&A, 326, 950

Foley, R. J., et al. 2009, AJ, 137, 3731

Gallagher, J. S., Garnavich, P. M., Berlind, P., Challis, P., Jha, S., \& Kirshner R. P. 2005, ApJ, 634, 210

Gallagher, J. S., Garnavich, P. M., Caldwell, N., Kirshner, R. P., Jha, S. W., Li, W., Ganeshalingam, M., \& Filippenko, A. V. 2008, ApJ, 685, 752

Garnavich, P. M., et al. 2004, ApJ, 613, 1120

Gil de Paz, A., et al. 2007, ApJS, 173, 185

Guy, J., Astier, P., Nobili, S., Regnault, N., \& Pain, R. 2005, A\&A, 443, 781

Hamuy, M., Phillips, M. M., Maza, J., Suntzeff, N. B., Schommer, R. A., \& Aviles, R. 1995, AJ, 109, 1

Hamuy, M., Trager, S. C., Pinto, P. A., Phillips, M. M., Schommer, R. A., Ivanov, V., \& Suntzeff, N. B. 2000, AJ, 120, 1479
Hicken, M., Challis, P., Jha, S., Kirshner, R. P., Matheson, T., Modjaz, M., Rest, A., \& Wood-Vasey, W. M. 2009a, ApJ, 700, 331

Hicken, M., Wood-Vasey, W. M., Blondin, S., Challis, P., Jha, S., Kelly, P. L., Rest, A., \& Kirshner, R. P. 2009b, ApJ, 700, 1097

Howell, D. A. 2001, ApJ, 554, L193

Howell, D. A., Sullivan, M., Conley, A., \& Carlberg, R. 2007, ApJ, 667, L37

Howell, D. A., et al. 2009, ApJ, 691, 661 (H09)

Jha, S., Riess, A. G., \& Kirshner, R. P. 2007, ApJ, 659, 122

Kasen, D. 2006, ApJ, 649, 939

Kobayashi, C., \& Nomoto, K. 2007, ApJ, in press, arXiv:0801.0215

Le Borgne, D., \& Rocca-Volmerange, B. 2002, A\&A, 386, 446

Le Borgne, D., Rocca-Volmerange, B., Prugniel, P., Lançon, A., Fioc, M., \& Soubiran, C. 2004, A\&A, 425, 881

Lee, H., Skillman, E. D., Cannon, J. M., Jackson, D. C., Gehrz, R. D., Polomski, E. F., \& Woodward, C. E. 2006, ApJ, 647, 970

Martin, D. C., et al. 2005, ApJ, 619, L1

Parodi, B. R., Saha, A., Sandage, A., \& Tammann, G. A. 2000, ApJ, 540, 634

Perlmutter, S., et al. 1997, ApJ, 483, 565

Perlmutter, S., et al. 1999, ApJ, 517, 565

Phillips, M. M. 1993, ApJ, 413, L105

Prieto, J. L., Rest, A., \& Suntzeff, N. B. 2006, ApJ, 647, 501

Prieto, J. L., Stanek, K. Z., \& Beacom, J. F. 2008, ApJ, 673, 999

Riess, A. G., Press, W. H., \& Kirshner, R. P. 1996, ApJ, 473, 88

Riess, A. G., et al. 1998, AJ, 116, 1009

Schawinski, K. 2009, MNRAS, 397, 717

Schlegel, D. J., Finkbeiner, D. P., \& Davis, M. 1998, ApJ, 500, 525

Sullivan, M. 2009, in AIP Conf. Proc. 1111, Probing Stellar Populations Out to the Distant Universe: CEFALU 2008, ed. L. A. Antonelli et al. (Melville, NY: AIP), 539

Sullivan, M., Ellis, R. S., Howell, D. A., Riess, A., Nugent, P. E., \& Gal-Yam, A. 2009, ApJ, 693, L76

Sullivan, M., et al. 2006, ApJ, 648, 868

Taubenberger, S., et al. 2008, MNRAS, 385, 75

Timmes, F. X., Brown, E. F., \& Truran, J. W. 2003, ApJ, 590, L83

Tonry, J. L., et al. 2003, ApJ, 594, 1

Tremonti, C. A., et al. 2004, ApJ, 613, 898

Tripp, R. 1998, A\&A, 331, 815

Tripp, R., \& Branch, D. 1999, ApJ, 525, 209

Truran, J. W., Arnett, W. D., \& Cameron, A. G. W. 1967, Can. J. Phys., 45, 2315

Wood-Vasey, W. M., et al. 2008, ApJ, 689, 377

York, D. G., et al. 2000, AJ, 120, 1579 